\title{
The T2K ND280 Off-Axis Pi-Zero Detector
}

S. Assylbekov ${ }^{\mathrm{b}}$, G. Barr ${ }^{\mathrm{d}}$, B.E. Berger ${ }^{\mathrm{b}}$, H. Berns ${ }^{\mathrm{h}}$, D. Beznosko ${ }^{\mathrm{c}}$, A. Bodek $^{\mathrm{f}}$, R. Bradford $^{\mathrm{f}, 1}$, N. Buchanan ${ }^{\mathrm{b}}$, H. Budd $^{\mathrm{f}}$, Y. Caffari $^{\mathrm{b}}$, K. Connolly ${ }^{\text {, I. Danko }}$, R. Das ${ }^{\mathrm{b}}$, S. Davis ${ }^{\mathrm{h}}$, M. Day ${ }^{\mathrm{f}}$, S. Dytman ${ }^{\mathrm{e}}$, M. Dziomba ${ }^{\mathrm{h}}$, R. Flight ${ }^{\mathrm{f}}$, D. Forbush ${ }^{\mathrm{h}}$, K. Gilje ${ }^{\mathrm{c}}$, D. Hansen ${ }^{\mathrm{e}}$, J. Hignight $^{\mathrm{c}}$, J. Imber ${ }^{\mathrm{c}}$, R.A. Johnson ${ }^{\mathrm{a}}$, C.K. Jung ${ }^{\mathrm{c}}$, V. Kravtsov ${ }^{\mathrm{b}}$, P.T. Le ${ }^{\mathrm{c}}$, G.D. Lopez ${ }^{\mathrm{c}}$, C.J. Malafis ${ }^{\mathrm{c}}$, S. Manly ${ }^{\mathrm{f}}$, A.D. Marino ${ }^{\mathrm{a}, *}$, K.S. McFarland ${ }^{\mathrm{f}}$, C. McGrew ${ }^{\mathrm{c}}$, C. Metelko ${ }^{\mathrm{g}}$, G. Nagashima ${ }^{\mathrm{c}}$, D. Naples ${ }^{\mathrm{e}}$, T.C. Nicholls ${ }^{\mathrm{g}}$, B. Nielsen ${ }^{\mathrm{c}}$, V. Paolone ${ }^{\mathrm{e}}$, P. Paul ${ }^{\mathrm{c}}$,

G.F. Pearce ${ }^{\mathrm{g}}$, W. Qian ${ }^{\mathrm{g}}$, K. Ramos ${ }^{\mathrm{c}}$, E. Reinherz-Aronis ${ }^{\mathrm{b}}$, P.A. Rodrigues ${ }^{\mathrm{f}}$, D. Ruterbories ${ }^{\mathrm{b}}$, J. Schmidt ${ }^{\mathrm{c}}$, J. Schwehr $^{\mathrm{b}}$, M. Siyad ${ }^{\mathrm{g}}$, J. Steffens $^{\mathrm{c}}$, A.S. Tadepallic ${ }^{\mathrm{c}}$ I.J. Taylor ${ }^{\mathrm{c}}$, M. Thorpe ${ }^{\mathrm{g}}$, W. Toki ${ }^{\mathrm{b}, \mathrm{c}}$, C. Vanek ${ }^{\mathrm{a}}$, D. Warner ${ }^{\mathrm{b}}$, A. Weber ${ }^{\text {d,g }}$, R.J. Wilkes ${ }^{\mathrm{h}}$, R.J. Wilson ${ }^{\mathrm{b}}$, C. Yanagisawa ${ }^{c, 2}$, T. Yuan ${ }^{\mathrm{a}}$

\author{
${ }^{a}$ University of Colorado at Boulder, Department of Physics, Boulder, Colorado, U.S.A \\ ${ }^{b}$ Colorado State University, Department of Physics, Fort Collins, Colorado, U.S.A. \\ ${ }^{c}$ State University of New York at Stony Brook, Department of Physics and Astronomy, Stony Brook, New York, U.S.A. \\ ${ }^{d}$ Oxford University, Department of Physics, Oxford, United Kingdom \\ ${ }^{e}$ University of Pittsburgh, Department of Physics and Astronomy, Pittsburgh, Pennsylvania, U.S.A. \\ ${ }^{f}$ University of Rochester, Department of Physics and Astronomy, Rochester, New York, U.S.A. \\ ${ }^{g}$ STFC, Rutherford Appleton Laboratory, Harwell Oxford, United Kingdom \\ ${ }^{h}$ University of Washington, Department of Physics, Seattle, Washington, U.S.A.
}

\section{N Abstract}

The pi-zero detector (PØD) is one of the subdetectors that makes up the off-axis near detector for the Tokai-to-Kamioka (T2K) long baseline neutrino experiment. The primary goal for the PØD is to measure the relevant cross-sections for neutrino interactions that generate $\pi^{0}$ 's, especially the cross-section for neutral current $\pi^{0}$ interactions, which are one of the dominant sources of background to the $v_{\mu} \rightarrow v_{e}$ appearance signal in T2K. The PØD is composed of layers of plastic scintillator alternating with water bags and brass sheets or lead sheets and is one of the first detectors to use Multi-Pixel Photon Counters (MPPCs) on a large scale.

Keywords: Neutrinos, Neutrino Oscillation, Long Baseline, T2K, J-PARC, Pi zero Detector

\section{Introduction}

The Tokai-to-Kamioka (T2K) experiment is a long-baseline neutrino oscillation experiment designed to probe the mixing of the muon neutrino with other neutrino species and to shed light on the neutrino mass scale. The T2K neutrino beam is generated using the the new high-intensity proton synchrotron at J-PARC, which has a Phase-I design beam power of 0.75 MW. T2K uses Super-Kamiokande [1] as the far detector to measure neutrino rates at a distance of $295 \mathrm{~km}$ from the beam production point, and near detectors to sample the unoscillated beam. The neutrino beam is directed $2.5^{\circ}$ away from the Super-

- Kamiokande detector and travels through the Earth's crust under Japan, as illustrated in Fig. 1 .

The T2K experiment [2] near detector complex (ND280), located $280 \mathrm{~m}$ from the start of the neutrino beam, contains the on-axis INGRID detector and an off-axis detector.

The off-axis detector, shown in Fig. 2, is situated at the same off-axis angle as Super-Kamiokande and contains the Pi-Zero detector (PØD) a plastic scintillator-based detector optimized for $\pi^{0}$ detection followed by a tracking detector comprising

\footnotetext{
${ }^{*}$ Corresponding author

Email address: alysia.marino@colorado.edu (A.D. Marino)

${ }^{1}$ Presently at Argonne National Laboratory, Argonne, Illinois, U.S.A

${ }^{2}$ Also at BMCC/CUNY, New York, New York, U.S.A.
}

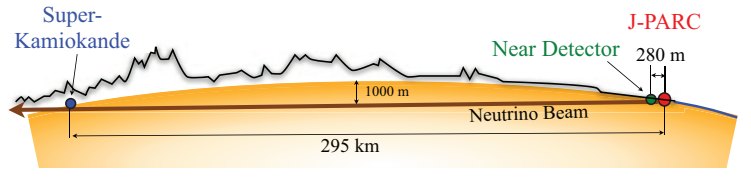

Figure 1: A schematic showing the path of the neutrinos in the T2K experiment, from the start of the neutrino beamline at J-PARC to Super-Kamiokande, 295 km away.

two fine grained scintillator detector modules (FGDs) sandwiched between three time projection chambers (TPCs). The PØD and tracker are surrounded by electromagnetic calorimeters (ECALs), including a module that sits immediately downstream of the tracker. The whole detector is located in a magnet with a $0.2 \mathrm{~T}$ magnetic field, which also serves as mass for a side muon range detector (SMRD). This paper describes the PØD subdetector in greater detail.

\subsection{Goals of the $P \emptyset D$}

The primary physics goal of $\mathrm{T} 2 \mathrm{~K}$ is to measure the mixing angle $\theta_{13}$ or to improve the existing limit by an order of magnitude if the angle is too small to measure directly. This is 


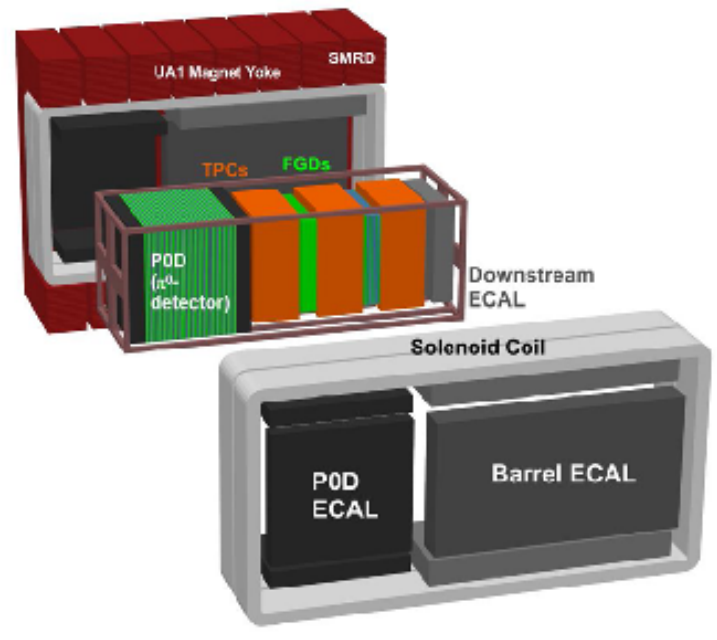

Figure 2: An exploded view of the off-axis near detector.

done by looking for the appearance of $v_{e}$ in a $v_{\mu}$ beam. Additional physics goals include the precision determination of the $\Delta m^{2}{ }_{23}$ and $\theta_{23}$ parameters through a $v_{\mu}$ disappearance measurement, where the parameters will be measured to a precision of $\delta\left(\Delta m^{2}{ }_{23}\right) \sim 10^{-4} \mathrm{eV}^{2}$ and $\delta\left(\sin ^{2} 2 \theta_{23}\right) \sim 0.01$ respectively. In addition to neutrino oscillation studies, the T2K neutrino beam (a narrow-band beam with a peak energy of about $600 \mathrm{MeV}$ ) will enable a rich physics program of neutrino interaction studies at energies covering the transition between the quasi-elastic and resonance production regimes.

To achieve the required precision for the $v_{e}$ appearance measurement (observed via the process $v_{e}+n \rightarrow e^{-}+p$ ), the neutral current $\pi^{0}$ rate $\left(v_{\mu}+N \rightarrow v_{\mu}+N+\pi^{0}+X\right)$ must be measured at the J-PARC site near the neutrino beam production point using the off-axis near detector. Events containing $\pi^{0}$ 's are the dominant physics background to the $v_{e}$ appearance signal at SuperKamiokande. The PØD sits at the upstream end of the off-axis detector and has been designed to precisely measure the neutral current process $v_{\mu}+N \rightarrow v_{\mu}+N+\pi^{0}+X$ on a water $\left(\mathrm{H}_{2} \mathrm{O}\right)$ target. In addition the PØD will constrain the intrinsic $v_{e}$ content of the beam flux which is an irreducible background to the $v_{e}$ appearance measurement.

Early design studies demonstrated that understanding the $\pi^{0}$ and $v_{e}$ backgrounds required sensitivity to interactions containing $\pi^{0}$ with momentum greater than $200 \mathrm{MeV} / c$. This requires a photon reconstruction threshold of well below $100 \mathrm{MeV}$. Both of the background processes to be constrained by the PØD are a relatively small fraction of the total PØD interaction rate, and must be measured on a water target, forcing a large water mass. In addition, sufficient energy resolution is needed to demonstrate the presence of a $\pi^{0}$ through reconstruction the invariant mass. The eventual design of the PØD realizes these goals by interleaving water target between scintillator layers which both measure charged particles and support the water target. The rate on water is determined using statistical subtraction with data collected during periods having water in the detector and out of the detector.

\subsection{Description of the $P \emptyset D$}

The main features of the PØD design are shown in Fig. 3 The electronics supports and detector mounting system are visible surrounding the active regions of the detector. In addition the regions of the detector are also labeled. Figure 4 shows a schematic of the active regions of the PØD. The central region, composed of the "upstream water target" and "central water target," is made from alternating scintillator planes, water bags, and brass sheets. The front and rear sections, the "upstream ECal" and "central ECal" respectively, use alternating scintillator planes and lead sheets. This layout provides effective containment of electromagnetic showers and a veto region before and after the water target region to provide rejection of particle interactions that enter from outside the PØD.

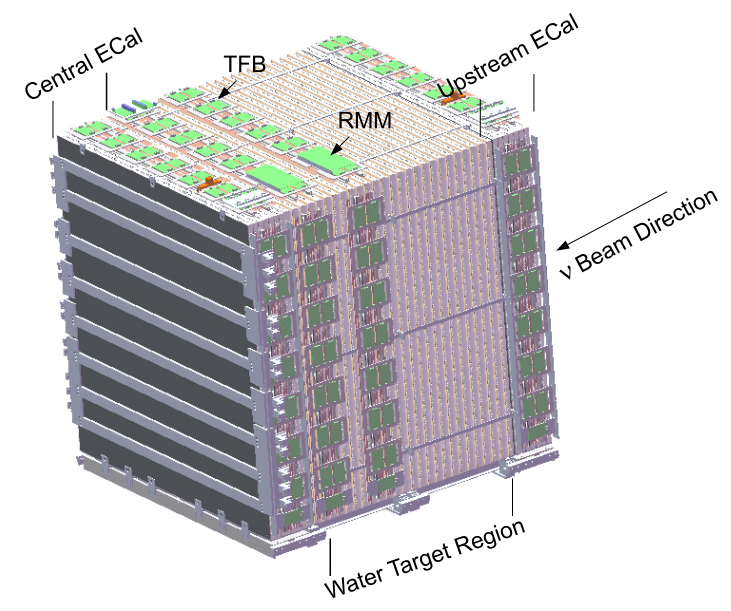

Figure 3: 3D drawing of the roughly $2.5 \mathrm{~m}$ cube PØD outside of the basket. Downstream face of detector shown. See Section 5 for a description of the TFB and RMM electronics.

There are a total of 40 scintillator modules in the PØD. Each PØD module, or PØDule, has two perpendicular arrays of triangular scintillator bars, forming a plane. There are 134 horizontal bars (2133 mm long) and 126 vertical bars $(2272 \mathrm{~mm}$ long) in each PØDule. Each bar has a single coaxial hole

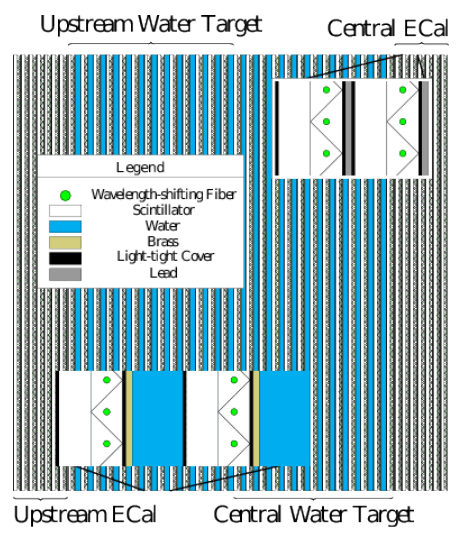

Figure 4: A schematic of the four PØD Super-PØDules as installed in the detector. Beam direction: left to right. 


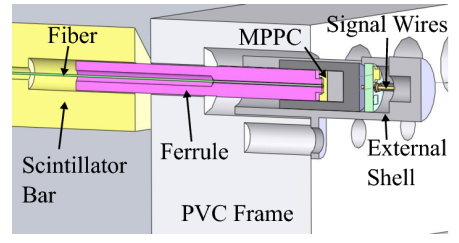

Figure 5: A close-up view of the edge of a PØDule showing how the WLS fibers exit the scintillator bars and couple to the MPPCs. The optical connectors will be described on more detail in Section 2.2.5

through which is threaded a wavelength-shifting (WLS) fiber. Each fiber has a mirrored coating applied on one end while the other end is optically coupled to a Hamamatsu multi-pixel photon counter (MPPC) [3] for readout, as shown in Figure 5] Each photodetector is read out with Trip-t Front-end electronics (Section 5. There are a total of 10,400 channels for the entire PØD.

The PØDules were assembled into four units called SuperPØDules. The two ECal Super-PØDules each consist of a sandwich of seven PØDules alternating with seven stainless steelclad lead sheets ( $4.5 \mathrm{~mm}$ thick). The water target is formed from two units, the upstream and central water target SuperPØDules. The upstream (central) water target Super-PØDule comprises 13 PØDules alternating with 13 (12) water bag layers (each of which is $28 \mathrm{~mm}$ thick), and 13 (12) brass sheets (1.28 $\mathrm{mm}$ thick), as shown in Fig. 6 The dimensions of the entire PØD active target are $2103 \mathrm{~mm} \times 2239 \mathrm{~mm} \times 2400 \mathrm{~mm}$ (width $\times$ height $\times$ length) and the mass of the detector with and without water is $15,800 \mathrm{~kg}$ and $12,900 \mathrm{~kg}$ respectively. The PØD is housed inside a detector basket structure that supports the central off-axis detectors inside the magnet.

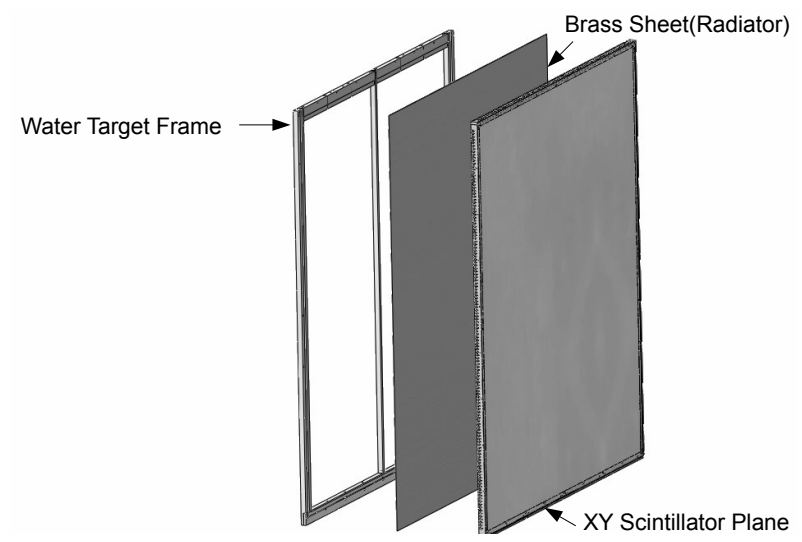

Figure 6: Expanded view of water target PØDule, brass radiator and water bladder containment frame.

The remainder of this paper describes in detail the design, fabrication, and performance of the PØD. The production of the scintillator bars and their assembly into planks and PØDules will be presented followed by a description of how the individual PØDules were combined into the four Super-PØDules, and are read out using photosensors. The detector component performance, starting with scans of the PØDules using a radioactive source, dark noise measurements, and tests with the light injection system, is presented. The paper concludes with a de- scription of the calibration and performance of the full detector.

\section{Design and Construction of the PØDule}

The PØDule is the basic structural element of the PØD active region, and is constructed of scintillator bars sandwiched between sheets of high-density polyethelene (HDPE, thickness $6.4 \mathrm{~mm}$ ). The entire structure is surrounded by PVC frames that support the PØDule as well as providing mounts for the required services such as the MPPC light sensors, and the light injection system.

The polystyrene triangular scintillating bars that make up the PØDules were fabricated by co-extruding polystyrene with a reflective layer of $\mathrm{TiO}_{2}$ and a central hole for the WLS fiber. The light seal for the tracking plane is maintained by light manifolds that collect the WLS fibers into optical connectors. These manifolds also provide access to the fibers for the light injection system. Because of the large number of scintillating bars and the available space limitations, it was impractical to route the fibers outside the magnetic volume therefore the Hamamatsu MPPC photosensors, which are immune to the magnetic field, were attached directly to each WLS fiber just outside the PVC PØDule frame, as shown in Figure 5.

\subsection{Design of the PØDule}

The PØDule was designed to both provide the active tracking region and to serve as a structural element. This was achieved using a laminated structure of crossed scintillator bars between polystyrene skins. The final PØDule has been shown to have a rigidity similar to a solid mass of polystyrene of similar thickness. The edge of the central scintillator and skin structure of the PØDule is surrounded by a machined PVC frame. Each PØDule is instrumented on one side (both $y$ and $x$ layers) with MPPCs and on the other a UV LED light injection system. The bottom PVC frame supports the weight of the PØDule within the ND280 detector basket. The frames also provide the fixed points needed to assemble the PØDule into the four SuperPØDules via two precision holes located in the four corners of each PØDule as well as a set of seven holes spaced along each side through which tensioning rods were passed.

The PØDules, after installation into the finished PØD, are oriented such that the most upstream layer of scintillator has the bars oriented approximately along the vertical axis while the downstream layer has its bars oriented along the horizontal axis. This arrangement results in a local coordinate system defined such that the $x, y$ and $z$ axes are approximately congruent with the global coordinate system where $x$ is horizontal, $y$ is vertical, and $z$ points downstream toward Super-Kamiokande. The external dimensions of the PØDule are $2212 \mathrm{~mm}(x)$ by $2348 \mathrm{~mm}(y)$ by $38.75 \mathrm{~mm}(z)$.

To facilitate assembly of the PØDule (described in Section 2.3, all of the components were prefabricated with holes that allowed alignment during assembly. The assembly tolerance was less than $0.5 \mathrm{~mm}$ on all internal dimensions, and less than $1 \mathrm{~mm}$ on the thickness. The relative dimensions of the PØDules were maintained using precisely located holes in the PØDule assembly table. 


\subsection{Assembly of PØDule Components}

The construction and assembly of the PØDule components was distributed across several institutions. This allowed a supply chain that could produce the required components in parallel and optimized use of facilities, local expertise and available personnel.

\subsubsection{PØDule Scintillator Preparation}

The polystyrene scintillator for the PØDule was manufactured in the extrusion facility at Fermi National Accelerator Laboratory [4] using an extrusion die originally developed for the inner detector of the MINER $v \mathrm{~A}$ experiment [5, 6]. The blue-light emitting scintillator base material was Dow Styron $663 \mathrm{~W}$ doped with $1 \%$ PPO and $0.03 \%$ POPOP to shift the UV scintillation light emitted by the polystyrene into the blue. The bars are triangular in cross section with nominal dimensions of $17 \pm 0.5 \mathrm{~mm}$ height and $33 \pm 0.5 \mathrm{~mm}$ width. Each bar also had a nominal $2.6 \mathrm{~mm}$ diameter hole centered at $8.5 \pm 0.25 \mathrm{~mm}$ above the widest part of the triangle for fiber insertion. To reflect the produced light and therefore maximize the possibility of capture by the wavelength shifting fiber in the center hole, a thin, $0.25 \mathrm{~mm}$ on average, layer of polystyrene mixed with $25 \%$ $\mathrm{TiO}_{2}$ was coextruded on the outside of the bar, and both ends of the scintillator bar were painted with white EJ-510 $\mathrm{TiO}_{2}$ Eljen paint.

During production, physical characteristics of the scintillator were monitored by taking frequent samples and measuring their outer dimensions, the location and dimensions of the center hole, and the thickness and coverage of the coextrusion. At approximately twenty different equally spaced times during production, samples were also taken and used to characterize light output using a radioactive source counting setup with a reference piece of scintillator from the MINER $v$ A production. Physical dimensions were held well within the tolerance, and no evidence was observed for variation in light output beyond the uncertainties in the monitoring measurement, roughly $5 \%$.

\subsubsection{PØDule Plank Assembly}

The extruded scintillator bars were bundled into manageable sized "planks" to be used in the assembly of the full-sized PØDules. There were two sizes of planks for each of the bar lengths and a special jig was constructed for each of the four plank types.

Each of the triangular scintillating bars was prepared for the plank assembly and subjected to quality assurance (QA) procedures prior to assembly into a plank. As each bar was unpacked it was inspected for signs of visible damage, such as nicks or cuts in the $\mathrm{TiO}_{2}$ coating and any damaged bars were removed from plank production. Once a bar passed the visual inspection it was cut to length using a jig to ensure proper length. A mounted pneumatic drill was used to bore out the ends of the holes running down the center of each bar. A long stiff wire was passed through each bar to ensure that no debris was lodged in the hole that would prevent insertion of the WLS fiber. An additional check was made to ensure that the hole for the fiber was centered on the end of the triangular bar.
Four separate jigs were set up on two optical tables for the gluing of the bars into planks. The short bars were made into two types of planks, one type containing 16 bars and one type containing 17 bars. The long bars were made into 15 bar and 17 bar planks. Prior to application of the epoxy to the bars, the bars were placed in the jig and a heavy straight-edge was used to ensure that the thickness of bars were within the $0.25 \mathrm{~mm}$ tolerance of the nominal $17.25 \mathrm{~mm}$ plank thickness. A log document, or traveler, was kept with each plank during the entire assembly process. The traveler contained details such as the plank serial number, the identification number of all bars contained in the plank, and any measurements made on the plank during assembly and quality assurance.

Once the bars had been test fitted into the plank gluing jig, they were removed and epoxy was applied to each using an automated gluing machine (Fig. 7) that mixed the two epoxy parts and applied a steady stream of glue to two sides of the bar. The epoxied bars were placed back into the gluing jig and a vacuum sealed frame was used to apply pressure to the plank for about 2 hours while the epoxy set. A final QA inspection was made to ensure that the planks were within the thickness tolerance.

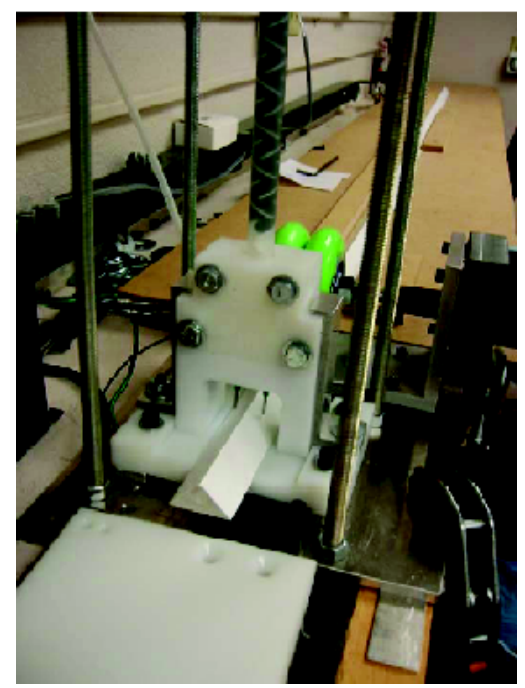

Figure 7: Automated glue machine used to apply epoxy to the triangular bars before they were placed into the plank gluing jig.

\subsubsection{PØDule WLS Fiber Preparation}

The wavelength shifting fibers that are inserted into the holes in the scintillator bars are Kuraray multi-clad, S-35, J-type, doped with Y-11 (175 ppm) with a diameter specification of $1.00_{-0.03}^{+0.02} \mathrm{~mm}$. The fibers were placed into the holes in the scintillator, but the hole was not filled with optical glue after the fiber was put into place. Studies done for the Minerva experiment indicated that the light yield for glued fibers was approximately 2 times greater than the light yield for unglued fibers [7], but unglued fibers are considerably easier to install. The same study also showed that the light yield did not strongly depend on the fiber-to-hole diameter ratio over the range of 0.3 to 0.9 [7], 
so the decision to use a $1.0 \mathrm{~mm}$ fiber in a $2.6 \mathrm{~mm}$ hole does not have a large impact on the light collection.

The WLS fiber was delivered in unspooled "canes" pre-cut to a rough length $67 \mathrm{~mm}$ longer than the bar length in order to avoid memory effects of spooled fiber.

The first step in processing the delivered fiber was to mirror one end. This work was performed in the Thin Film Coatings facility in Lab 7 at Fermi National Accelerator Laboratory. One end of the fiber was first "ice polished" with the ice providing mechanical support for a group of approximately 800 fibers polished with a diamond polisher in a single batch. The polished end was then coated with aluminum using a sputtering vacuum deposition process. After completion of the mirroring, each fiber was coated with a thin layer of epoxy to protect the mirror.

The reflectivity of three fibers from each batch of 800 was determined by measuring the light output of a fiber with the mirror end placed into a piece of scintillator with an attached radioactive source, and then remeasuring the light output after cutting off the mirror with a $45^{\circ}$ cleave and painting the cleaved end with black paint. For individual fibers, an average reflectivity of $86 \%$ was measured, with a root mean square of the ensemble of measurements of $6 \%$.

After mirroring, the opposite fiber end was glued into one end of a ferrule (see Section 2.2.5 and Figure 8) that was injection molded from Vectra A430, a Teflon-filled liquid-crystal polymer. The length between the ferrule end and the fiber mirror end was required to be kept at $1 \mathrm{~mm}$ tolerance over the several meter length of the fiber. The ferrule was designed to mount into a housing that contained the MPPC and kept the fiber end in contact with the pliable optical layer covering the MPPC. Because the Teflon in the Vectra plastic clogged the diamonds in the fiber polisher, the fiber was glued into the ferrule a few mm proud of the end of the ferrule, a $1 \mathrm{~mm}$ thick layer of optical epoxy was deposited for mechanical support, and then the epoxy with the embedded fiber was polished. The resulting finish was inspected with a microscope to establish when diamond wear adversely affected the finish. Typically, 1500 to 2000 fibers could be polished with a single diamond.

\subsubsection{MPPC Acceptance Testing}

The MPPCs used by the PØD, as well as by the ECAL, SMRD, and INGRID detectors, are solid-state photosensors manufactured by Hamamatsu Photonics. The active sensor for the MPPC is a $1.3 \mathrm{~mm} \times 1.3 \mathrm{~mm}$ array of 50 -micron pixels, totaling 667 pixels (including a small inactive area for electrical contact). Each pixel operates in Geiger mode, producing a well-defined pulse when a photoelectron generates a cascade. The MPPC output is the sum of all pixel outputs, the pixels having very similar responses. As a result, the output spectrum from the MPPC shows well-separated peaks corresponding to the number of pixels fired, which at low light levels is a good measure of the number of photoelectrons.

A photosensor quality-control (QC) testing program was performed on all 10,400 MPPCs installed in the PØD, as well as 1,100 spares. The goals of the testing were to measure the operational characteristics of the MPPCs, to verify that their perfor- mance was acceptable, and to set the initial operating voltages to be used in the PØD. In particular, the gain of each device was measured as a function of bias voltage. A linear fit was used to extract the breakdown voltage - the minimum bias voltage to produce a Geiger cascade. The MPPC characteristics were found to be largely consistent from device to device as a function of overvoltage (the bias voltage above the breakdown voltage), but there is a significant variation in breakdown voltages from device to device, particularly between devices originating in different batches. All tests were done at a controlled temperature of $20^{\circ} \mathrm{C}$, controlled to better than $0.2^{\circ} \mathrm{C}$, as the MPPC breakdown voltage depends on the operating temperature.

The testing protocol required the measurement of a number of different MPPC operating parameters. First, a scan of gain vs bias voltage was performed to measure the breakdown voltage and to establish the operating voltage. Measurements were made over a $2 \mathrm{~V}$ wide overvoltage range around the predicted gain range of $5 \times 10^{5}$ and $7.5 \times 10^{5}$. Dark noise rates and relative detection efficiencies at these gains were also measured.

Physics signals in the PØD range from a few to hundreds of photoelectrons. Eight light levels across this range were used to characterize the photosensors, with measurements made at four different bias voltages for each light level.

Production testing of the photosensors began in late September 2008 and was completed by January 2009. Photosensors passing all QC tests were shipped for installation into the completed PØDules. Only 230 out of 11,500 photosensors were rejected by the quality control procedure, despite stringent acceptance criteria. Of those rejected, 74 were broken during assembly, 76 were rejected due to dirt on the photosensor surface. Only 80 were rejected for abnormal behavior in the photosensor testing data.

A dedicated NIM paper describing the photosensor qualitycontrol testing and characterization procedure in more detail can be found at [8]. A paper describing the PØD MPPC testing in greater detail is under preparation.

\subsubsection{Optical Connectors}

Custom optical connectors were fabricated to provide optical fiber alignment to the MPPC active area and to reduce the signal rate due to light contamination from external sources to acceptable levels to well below the intrinsic dark noise of the MPPCs. The connector system, shown in Fig. 8 consists of three injection-molded components: a fiber-alignment tube or "ferrule", a housing that holds the MPPC and provides alignment to the fiber within $150 \mu \mathrm{m}$, and an external shell to provide mechanical protection and lock the connector on the ferrule. The material for all three molded components is VectraßA130, a $30 \%$ glass fiber loaded liquid-crystal polymer with very low shrinkage, excellent dimensional stability, and very good mechanical properties.

The MPPC is held in place against the fiber by a $3 \mathrm{~mm}$ thick closed-cell polyethylene foam disk, acting as a spring. To ensure good optical contact between fiber and MPPC, the fiber is allowed to protrude from the ferrule end by $0.5 \mathrm{~mm}$ after polishing, so the entire compression force from the foam spring is applied between the fiber end and MPPC face. Electrical 

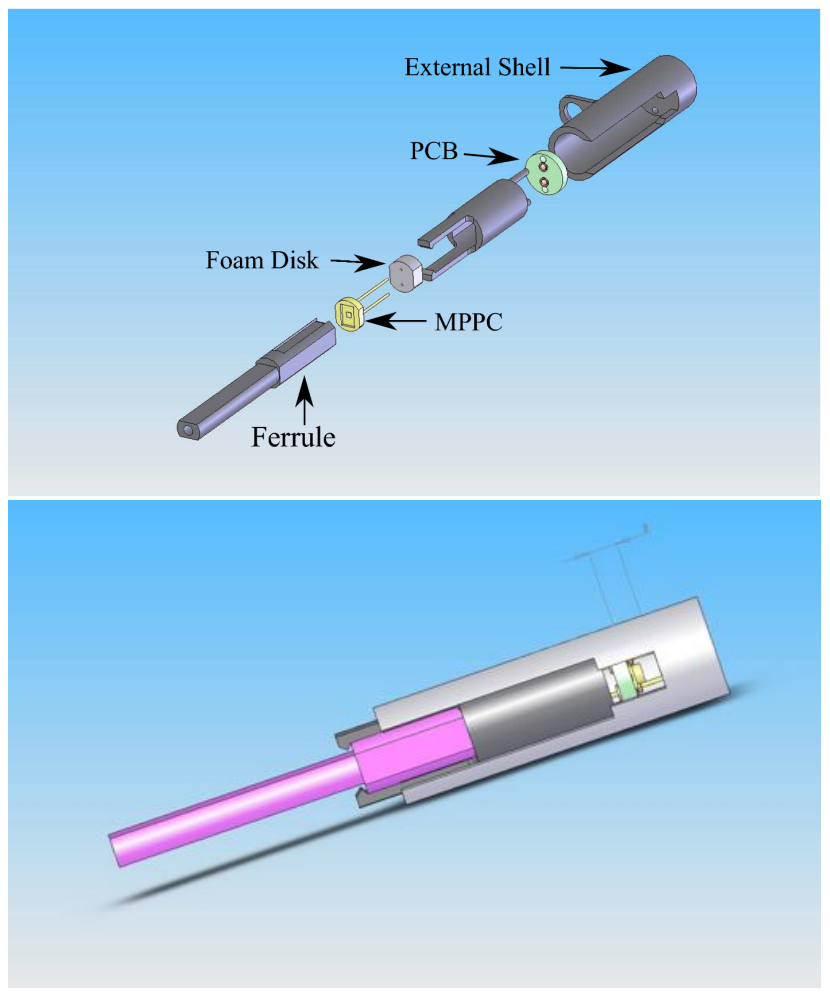

Figure 8: Optical connector: exploded (left) and assembled (right).

connection between the MPPC and the front-end electronics is provided via a small circular printed circuit board (PCB), with spring-loaded pin sockets making contact to the MPPC leads and a Hirose Electric Co. micro-coax connector.

Connectors of this type are used in ND280 for the P0D, ECAL and INGRID sub-detectors. Approximately 40,000 total connectors were produced (including spares).

\subsection{Assembly of the PØDule}

The PØDule assembly was one of the main fabrication stages to produce the forty PØDules (plus $10 \%$ spares). This step involved gluing the main mechanical components: the pre-glued scintillators planks, the four outer PVC frames, and the two outer HDPE plastic skins. A key requirement for this process was to keep within the tolerances of the thickness of the PØDule $(28 \mathrm{~mm})$ and to have the alignment of the scintillator bars match the wavelength shifting fiber holes in the scintillators with the holes in the PVC frames.

PØDule construction was performed on a specially designed gluing table. This flat table $(\sim 2.5 \times 2.5 \mathrm{~m})$ had precision alignment holes to keep the outer four PVC frames in the same location for all the PØDules. The first assembly step was the placement of the HDPE plastic sheet or skin on the gluing table, followed by painting HYSOL epoxy glue with paint roller brushes over the entire top side of the sheet. Next, the four outer PVC frames were placed on the edges of the glued sheet, and the position of the PVC frames were fixed by steel pins pressed into the holes in the table. Next, the scintillator $x$-layer planks were lowered onto the bottom HDPE sheet. They were aligned with respect to the PVC frame holes, then steel alignment pins were pressed through the frame holes and into the holes in the scintillator. After all the $x$-planks were pinned into place, epoxy glue was painted over the upper surface of the $x$-plank scintillator. Then the $y$-planks were lowered onto the epoxied $x$-planks, and again the $y$-plank positions were aligned with steel alignment pins. Next the upper HDPE outer sheet or "skin" was lowered on to the glue-painted y-plank surface.

After all the gluing was completed, a vacuum sheet was lowered onto the PØDule and vacuum was applied so the PØDule was uniformly compressed on all sides with about 0.5 atmosphere of pressure. The PØDule was left for about 12 hours to cure overnight. After the epoxy had cured, the vacuum sheet and the pins were removed and the PØDule was loaded with 260 WLS fibers that were each attached to the snap-on optical connector containing an MPPC. Finally the 260 MPPCs were connected via mini-coax cables to the scanner readout electronic boards. The PØDule was scanned overnight with a ${ }^{60} \mathrm{Co}$ source and read out as described in Section 6.2

During the peak of the construction phase, five PØDules were produced per week. After the PØDules were constructed and successfully scanned, they were stacked together vertically into the four Super-PØDules.

\subsection{The PØDule Light Injection System}

The purpose of the PØD Light Injection System (LIS) is to provide monitoring for all 10,400 channels in the PØD. The LIS is capable of exposing the MPPC photosensors to light intensities covering a range of more than two orders of magnitude with flash-to-flash intensity stability of less than $2 \%$ [9]. This allows for monitoring of the photosensor response over the full range of energy deposition expected for neutrino interactions in the PØD.

The main design challenge of the LIS was the geometrical constraint that it be embedded within the $3 \mathrm{~cm} \times 4 \mathrm{~cm} \times 220$ $\mathrm{cm}$ PØDule layer support frame for each of the 80 scintillator planes. Each PØDule support frame has a cavity that allows 5 mm segments of WLS fibers to be exposed to light produced by a pair of LEDs (Fig. 9). The LIS uses 80 pairs of fast-pulsed 400 nm UV LEDs as light sources; each pair illuminates a cavity in a single PØDule support frame. LEDs exhibit minimal pulseto-pulse fluctuations in intensity, so the temporal response of an MPPC photosensor is dominated by photoelectron statistics.

The LIS provides the capacity to vary the LED light intensity over the required dynamic range through control of both the height and the width of the pulses, by varying the current pulse applied to the LED. The LEDs are driven by electronics originally designed for the MINOS experiment LIS system [9]. The LIS electronics consists of four pulser boxes, a control box, a distribution box and a power supply. The pulser boxes are mounted on the bottom and the north sides on the PØD in close proximity to the LEDs. The control box is situated under the PØD, mounted on the detector basket. The distribution box and the power supply are located in a rack about 10 meters from the PØD. Each pulser box contains two LED driver boards, a controller board, and an LVDS to TTL converter board. Each driver 
board has ten channels that can be programmed to pulse simultaneously in group of of 5, 10, or 20 channels. In contrast to the MINOS light injection system, each channel is connected to a pair of LEDs by a $60 \mathrm{~cm}$ long shielded cable. Communication between a PIC16F877 microprocessor located on the controller board and a control computer is handled via ASCII commands over a serial RS232 link. Signals from the control computer are carried over an Ethernet link and converted for the pulser boxes by an Ethernet-RS232 converter.

During normal operation the PØD LIS control computer instructs the system to pulse a specific combination of LEDs at a specific height, width, and frequency to monitor the temporal behavior of the PØD and associated readout. Dedicated LIS runs are taken periodically to allow more precise measurements of properties, such as the relative timing between the PØD channels.

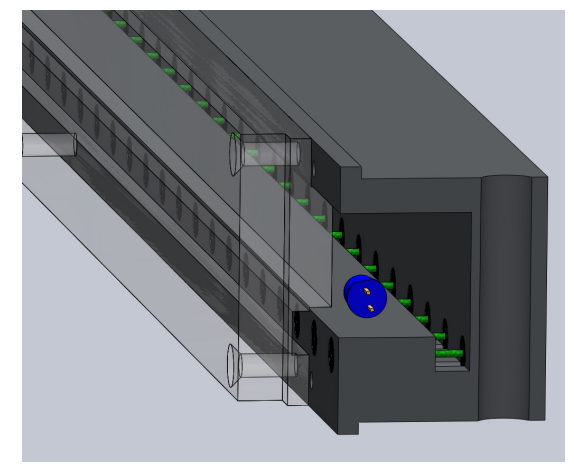

Figure 9: Cross-section view of the LIS cavity. Not shown are transparent strips with an opaque band to shadow nearby fibers.

\section{Water Targets}

\subsection{Water Target Design}

The water target modules were designed to provide layers of water approximately $3 \mathrm{~cm}$ thick in the beam direction, with transverse dimensions of approximately $2 \mathrm{~m} \times 2 \mathrm{~m}$. In order to provide such large but thin layers without excessive container mass, high-density polyethylene (HDPE) bladders were fabricated using materials and techniques derived from experience with the Pierre Auger Observatory Water Cherenkov detector liners [10]. Adjacent scintillator bar layers provided the structural support required, with water pressure transmitted through successive PØDules to end plates attached to the ND280 basket frame.

Since the water bladders are not rigid, it is necessary to protect against excessive deflection of adjacent scintillator bar layers due to unbalanced pressure, such as the case where one water layer is emptied while the others remain full. A vertical HDPE center strut to limit such deflections was required, so each water layer consists of two side-by-side bladders, each approximately $1 \mathrm{~m}$ wide by $2 \mathrm{~m}$ high by $3 \mathrm{~cm}$ thick.

The bladders were heat-welded and sealed into HDPE frames that provide ports for fill, drain, and sensor tubes. The frames also accommodate the mounting and support hardware used to assemble the Super-PØDules. The frames were edged with silicone sponge gasket material (Stockwell Elastomerics R10470), such that each bladder was effectively encased within a waterproof seal when compressed between adjacent PØDules. This provides a second level of water containment to protect against leaks. The water bladder frames were equipped with drain ports to direct any water captured within the gaskets into two drip pans mounted below the PØD and basket, one on either side of the basket centerline. Each target layer was filled with water and left overnight prior to acceptance and shipment for integration with scintillator layers and assembly into SuperPØDules. The upstream target Super-PØDule had 13 water layers (26 bladders) and the central target Super-PØDule had 12 layers ( 24 bladders), for a total of 25 water target layers and 50 bladders. After integration, water fill/drain testing was repeated and repeated a third time when the Super-PØDules arrived at J-PARC.

In order to meet the physics analysis requirements, a water pump and monitoring system was designed to allow individual bladders to be filled, drained, and also provide water depth data. A pump rack with 50 self-priming bellows pumps (GormanRupp Industries (GRI) 16001-005 F-009 T-007), one for each bladder, was designed with valves arranged to allow each pump to be used either to fill or drain a single bladder. The pump rack is located outside the magnet. The pumps are connected to fill and drain tubes on the bladders using Polyflo 66P 3/8-inch OD polyethylene tubing.

Water is supplied from a 3000 liter high-density polyethylene tank (DenHartog model VT0900-46), next to the pump rack, coupled to the pumps through two 20-liter buffer tanks on the rack. The main tank is filled with filtered tap water, with commercial chlorine bleach added as a biocide at an effective concentration of $0.025 \%$ sodium hypochlorite by volume.

Each bladder is equipped with separate drain and fill tubes made of 3/8-inch schedule 40 PVC pipe, and has two additional PVC pipes containing water monitoring sensors of two kinds: binary level sensors, which simply report their state as wet or dry, and depth (pressure) sensors.

\subsection{Fabrication of Water Targets}

The water target bags are made from a 1 meter wide continuous roll of seamless polyethylene plastic tubing. The plastic sheet is $0.1 \mathrm{~cm}$ thick, so the combined thickness of 2 walls of the water bag is $0.2 \mathrm{~cm}$. The tubing is cut to the length of the PØDule height, and is heat welded to make a bag with a leak proof seal at the bottom with no seams on the sides of the bag. Two HPDE frames are then attached, one on the top and one on the bottom section of the bag. The top frame is slotted to provide entrance holes from the top for the water tubes and the pressure and level sensor pipes. The top HDPE frame length is held in position by the HDPE gasket frames on the PØDule. The bottom frame is threaded to allow for through-screws, protruding from the bottom HDPE frame of the gasket frame, to pull the bag taut in the enclosure. This arrangement allows for a leaky bag to be pulled straight from the top of the water target 
Super-PØDule and replaced by lowering a new bag from the top without removing the Super-PØDule out of the basket frame.

\subsection{Instrumentation of the water targets}

In order to properly monitor the water level in each water target layer, sensors are inserted into each water target bladder. Accurate water level readings are needed not only for safety and engineering concerns, but also to determine the water mass inside the fiducial volume of the PØD to the desired accuracy of $3 \%$.

The monitoring system consists of sensors that are inserted into the bladder and operated in the water, an external monitoring sensor for the environment, and a DAQ system. The entire system is designed to be independent of the ND280 Global Slow Control system in order to be independent of shutdowns and to provide additional flexibility needed during PØD fill or drain operations.

Two sensor pipes are installed in each of the 50 water bladders. In each bladder, both pipes have a Global Water WL400 water depth sensor at the bottom end of the pipe. Each pipe also have one Honeywell LLE series Liquid Level Sensor binary wet-dry level sensors placed near its top, for calibration and back-up purposes. All new sensor pipes were water tested overnight or longer prior to deployment. The depth sensors are calibrated by filling test pipes to a series of measured heights, and logging repeated depth readings. These data are then used to fit calibration curves relating depth to current-loop mA. Results are consistent with factory calibration data. The calibration process is checked with a 1-point measurement using an identical test stand after shipment to J-PARC.

The water sensors required an auxiliary, custom built, connection board to distribute DC power and convert current-loop signals to voltage signals to be read out by the sensor boards. The sensor boards are mounted on the top of the PØD, above the water target bladders. This board contained a SenSym ICT series ASDX Pressure Transducer, a Texas Instruments TMP275 Digital Temperature Sensor, 12-bit ADCs to read the sensor outputs, and digital logic to communicate with the rest of the DAQ. Communication between the DAQ and system hardware components is performed using $\mathrm{I}^{2} \mathrm{C}$. Signals are converted to RS-232 serial data for transmission due to the distance between the PØD and the DAQ computer. Sensor signals were digitized on the Sensor Boards, which were in turn connected to a custom built, 8 port Multiplexer (Mux) Board using the $\mathrm{I}^{2} \mathrm{C}$ bus. A total of four Mux boards are required. The Mux Board converted the $\mathrm{I}^{2} \mathrm{C}$ signal to $\mathrm{RS} 232$ and also supplied power to the sensors and electronics. The sensors are controlled and monitored by a graphical interface using the Qt 4.0 application and user interface. The monitoring program controls the sensor readout, interprets the data, feeds the data to the GSC system, and then stores the data locally.

An identical WL400 sensor and readout board is installed in the main water storage tank in order to monitor its water level during filling and draining.

\begin{tabular}{|l|c|c|c|}
\hline Super-PØDule & $\begin{array}{c}\text { Mass } \\
(\mathrm{kg})\end{array}$ & $\begin{array}{c}\text { Dimensions } \\
(\mathrm{mm} \times \mathrm{mm} \times \mathrm{mm})\end{array}$ & $\begin{array}{c}\text { Depth } \\
\text { in R.L. }\end{array}$ \\
\hline Upstream ECal & 2900 & $2298 \times 2468 \times 305$ & 4.946 \\
Upstream Water & & $2298 \times 2468 \times 888$ & \\
Target: & 3600 & & \\
Empty & 5100 & & 1.370 \\
Filled & & $2298 \times 2468 \times 854$ & \\
Central Water & & & \\
Target: & 3500 & & 1.356 \\
Empty & 4900 & & 2.287 \\
Filled & 2900 & $2298 \times 2468 \times 304$ & 4.946 \\
Central ECal & & & \\
\hline
\end{tabular}

Table 1: The mass, dimensions, and depth in radiation lengths for each SuperPØDule.

\section{Super-PØDules}

The full PØD detector is constructed of four Super-PØDules assembled from PØDules. Figure 4 shows the arrangement of the four Super-PØDules in the assembled detector as well as details of the structure within each type of Super-PØDule.

\subsection{The Super-PØDule Design and Tooling}

The four Super-PØDules that make up the PØD were designed to simplify the installation and shipping from the assembly site to the installation site at J-PARC while allowing for efficient assembly. This was achieved by assembling all four modules from a set of standardized components (PØDules, water target modules, brass radiators, and lead radiators) that could be preassembled prior to the Super-PØDule assembly. The components were assembled onto their final mounting hardware, which was held on custom rolling carts. The carts were designed to support the Super-PØDules and allow them to be moved. After arrival in Japan, the Super-PØDules were kept on their individual carts which allowed them to be moved and tested independently.

Table 1 shows the mass, dimensions and the depth in radiation lengths of each Super-PØDule. During assembly the component masses were sampled and used to estimate the dry mass of each Super-PØDule. The mass of the water added to the target Super-PØDule is accounted for separately. We estimate that the dry mass uncertainty is approximately $0.8 \%$. The length of each Super-PØDule along the neutrino beam axis was measured after assembly and is estimated to have an accuracy of $0.5 \mathrm{~mm}$. The width and height dimensions of the SuperPØDule are perpendicular to the beam axis and have a tolerance of $5 \mathrm{~mm}$. These dimensions include space for the TFB read out electronics.

\subsection{ECal Super-PØDule Assembly}

An ECal Super-PØDule is assembled from seven PØDules interleaved with seven layers of lead plates. The assembly began with the construction of the lead panels. The first step was the preparation of a $0.05 \mathrm{~cm}$ thick, or 0.03 radiation lengths 
(R.L.), stainless steel (S.S.) sheet which was mounted on a flat assembly table. A thin aluminum frame was then epoxied and screwed on all four sides of the sheet. Epoxy was applied to the surface of the S.S. sheet and $253.45 \mathrm{~mm}$ thick (0.67 R.L.) lead strips were gently positioned side-by-side onto the epoxied surface. The lead plates were precut to size to minimize any gaps. The lead plates were then painted with epoxy and two stainless steel half-width sheets were placed onto the preepoxied lead plates, creating a panel that contained lead plates sandwiched between a pair of S.S. sheets. A vacuum cover was placed over the entire assembly and evacuated prior to allowing the epoxy to set overnight. Figure 10 is a photo of a S.S. plate being placed onto a layer of lead panels that have been painted with black epoxy.

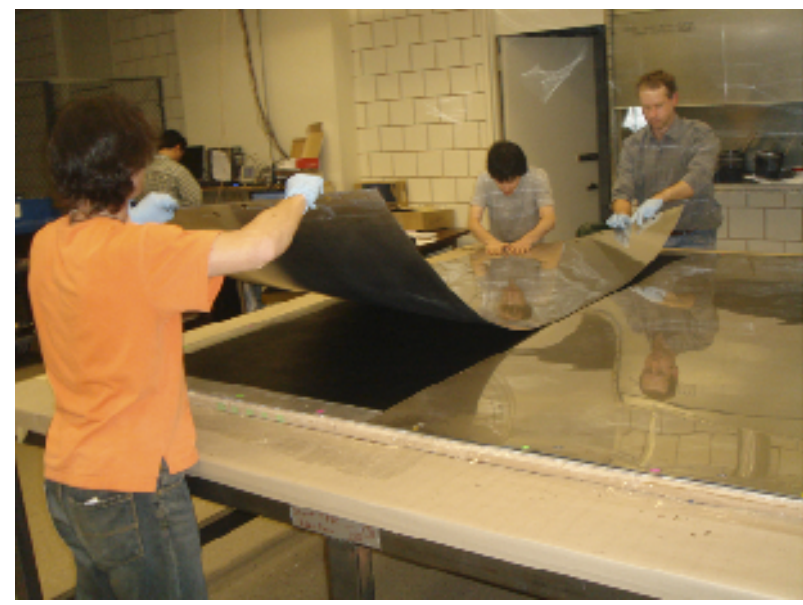

Figure 10: A stainless steel plate being placed onto a lead panel

Once the epoxy had set overnight, a U-channel beam was placed across the top of the completed lead panel and screwed into position. The U-channel beam was then lifted with a forklift to position the lead panel and rotated to hang vertically. The lead panel was then moved to a rollabout cart and mounted to a frame. Once the panel was positioned and held into place by the cart frame, the U-channel beam was removed. The completed PØDule, was then lifted vertically and mounted onto the lead panel. This process was repeated seven times to complete an ECal Super-PØDule. After the seventh PØDule was added, threaded stainless steel rods were screwed through clear holes around the periphery of the PØDules and secured in place with a special flat nut.

Finally the electronics mounting rail assemblies were attached to the top and on one side of the ECal Super-PØDules where the MPPCs were mounted. Once the readout electronics (see Section 5) were installed, the mini-coax cables from 1820 MPPCs were connected to the TFBs and labeled, completing the assembly. Figure 11 is a photo of a fully assembled PØDule, mounted vertically on a rollabout stand.

\subsection{The Water Target Super-PØDule Assembly}

The water target bag Super-PØDule assembly on the PØDules consisted of two water bladders that were mounted

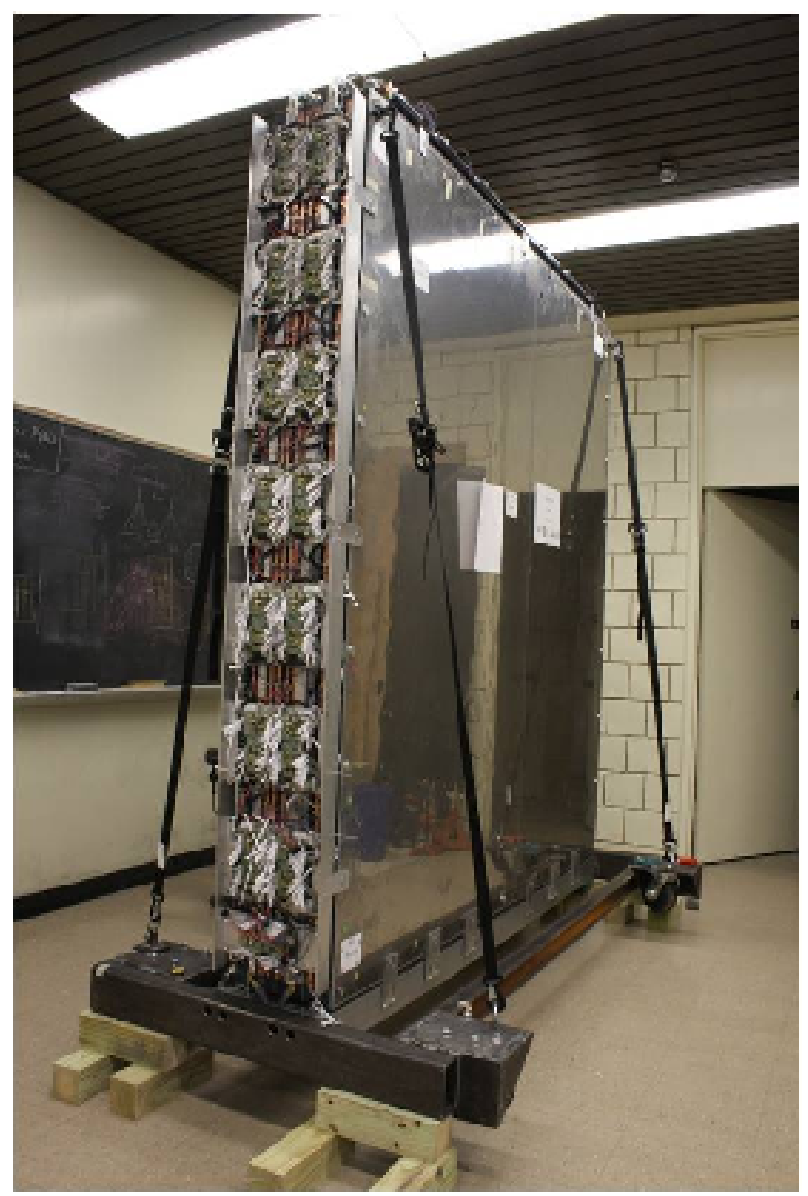

Figure 11: The fully-assembled upstream ECal Super-PØDule including the TFB readout electronics boards.

on the face of one PØDule and encased by HDPE frames on four sides (top, bottom, left, and right). This procedure was performed on 25 PØDules to form two separate water target Super-PØDules that held 12 or 13 water layers.

The assembly began with a PØDule placed flat on an assembly table. Two brass sheets of thickness, $0.15 \mathrm{~cm}$ (0.1 R.L.) were placed on the PØDule. A thin weather strip gasket was placed along the PØDule edges on two sides and the bottom. Three HDPE frames were then placed over the weather strips to form a water tight gasket seal and then a vertical HDPE center divider strip was placed in the middle of the PØDule. Next, the two water bladders were placed on the brass sheet. Each water bag had a top and bottom HDPE frame. The next step was to place the weather strip gasket in a groove in the three HDPE frames.

Finally a thin HDPE cover sheet was placed over the entire surface to form a water-tight seal and to keep the water bladders from protuding out when the PØDule was mounted vertically and moved. The upper water bladder HDPE frame was supported by slots in the upper HDPE gasket frame. The lower water bladder HDPE frame had mounting holes that were screwed down with screws extending through the bottom HDPE gasket frame. The bottom HDPE gasket frame had drain holes or ports, which were connected to a drain hose to allow any water leak 
to drain out in a controlled manner.

\subsection{Shipping and Installation of the Super-PØDules}

Each of the four Super-PØDules was mounted on a solid wooden base and enclosed in a wooden crate for shipping. The four Super-PØDules were flown from JFK airport in New York to the Narita International Airport near Tokyo by the Nippon Express shipping company in April 2009. The crated SuperPØDules were then delivered to J-PARC on two flatbed trucks and offloaded into the LINAC building where they were unpacked, removed from their wooden bases and checked out in preparation for installation. All electrical and plumbing utilities were installed in the detector hall and attached to the off-axis detector basket while the Super-PØDules were being checked out in the LINAC building, which simplified the installation of the Super-PØDules into the basket.

Each Super-PØDule remained bolted to custom rolling carts until just prior to installation. Aluminum covers were attached to the side and top electronics of the Super-PØDule to prevent damage during installation into the detector basket. SuperPØDules were installed in order from the most upstream side of the detector basket. For installation each Super-PØDule was disconnected from its cart and lifted by a crane using a custom lift fixture (Fig. 12) that allowed precise positioning of the Super-PØDule as it was lowered into the basket about $20 \mathrm{~m}$ below the staging area. Figure 12 shows the first Super-PØDule, the upstream ECAL, being lowered into position with guidance from a local contractor.

Once a Super-PØDule was set into position in the basket, it was bolted into place and utility connections were made to it. Precise positioning of each Super-PØDule in the basket was accomplished using adjustment screws. Following the utility connections and the filling of the water targets, aluminum covers were attached to the PØD in preparation for data taking.

\section{Readout Electronics and DAQ}

The PØD, like the SMRD, ECal, and INGRID detectors in the ND280, uses Trip-t-based front-end electronics [11] to read out its 10,400 photosensors. Each Trip-t Front-end Board (TFB) contains four 32-channel Trip-t ASIC chips [12], originally developed at Fermilab for the $\mathrm{D} \varnothing$ experiment, and can serve up to 64 MPPC sensors, which are connected by miniature coaxial cables to the TFB. The signal from the photosensor is divided capacitively in the ratio of 10:1 and routed into separate Trip-t channels to provide a high and a low gain response to the same input, thereby increasing the dynamic range of the electronics. The high gain channel provides measurement for up to a 50 photoelectron (p.e.) signal with $\sim 10$ ADC/p.e. resolution, while the low gain channel can be used to measure larger signals up to about 500 p.e.

The Trip-t chips integrate the charge in 23 consecutive integration cycles that are synchronized with the accelerator beam spill such that each $58 \mathrm{~ns}$ wide beam bunch, separated by $\sim 580$ $\mathrm{ns}$, falls into a separate integration window 100 ns away from the start of each integration cycle. The length of the integration

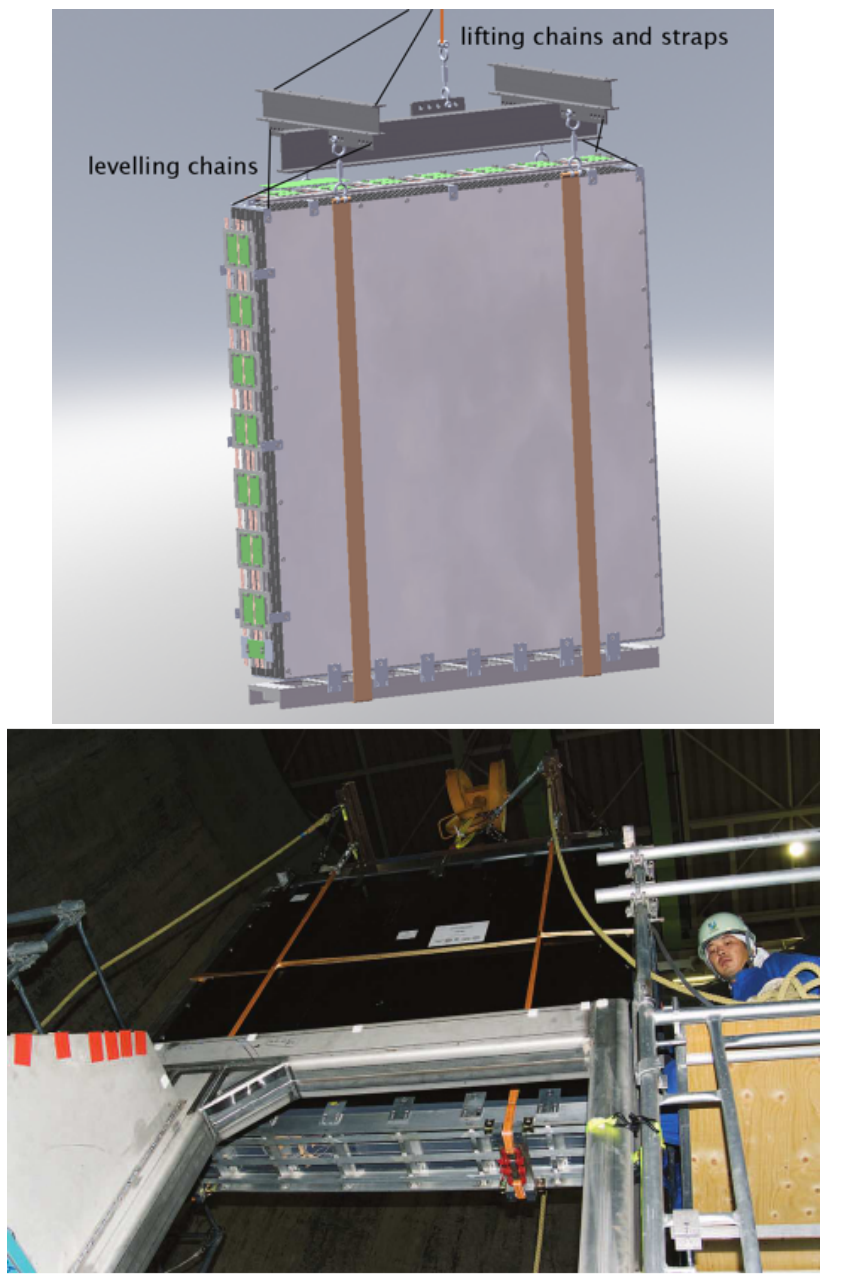

Figure 12: The left figure shows an engineering drawing of an ECAL module being lifted by the custom lift fixture, while the right picture shows the first Super-PØDule (upstream ECAL) being lowered into the detector basket. 
cycle and the reset period between them can be programmed and are set to $480 \mathrm{~ns}$ and $100 \mathrm{~ns}$ respectively for beam operation. These values will virtually eliminate deadtime for in beam spill interactions but will result in some deadtime for out of spill events such as Michel electrons. The Trip-t stores the results of the 23 integration cycles in its analog pipeline, which are digitized using a 10-bit ADC when the data are transferred off the board following the end of the integration period. The high gain channels also feed an internal discriminator that measures the time when the integrated signal exceeds a programmable preset threshold of about 2.5 p.e. in each integration cycle. The operation of the TFB is controlled by a Field Programmable Gate Array (FPGA), which also provides time stamping for the discriminator output with a $2.5 \mathrm{~ns}$ resolution, and moves the data from the Trip-t to the back-end electronics.

The TFB operation requires four low voltage levels. The bias voltages for the MPPCs are also provided through the TFB with the central core of the mini-coax cables connecting the sensors to the board being at the same uniform voltage level $(\sim 70 \mathrm{~V})$. Individual voltage adjustments are achieved by setting the voltage level of the shield sheath of the coax cable between 0 to 5 $\mathrm{V}$ in $20 \mathrm{mV}$ increments using an 8-bit DAC.

The entire PØD readout requires 174 TFBs; 29 for each of the upstream and central ECal Super-PØDules and 58 for each of the water target Super-PØDules. The back-end electronics of the PØD consist of 6 read-out merger modules (RMM), a cosmic trigger module (CTM) shared with the SMRD and the downstream ECal (DsECal), a slave clock module (SCM), and one master clock module (MCM) for the whole ND280 detector. Each RMM serves as a communication interface between the 29 TFBs and the data acquisition (DAQ) system, by passing control commands, clock, and trigger signal in one direction, and data in the other. Signals between the RMM and TFBs are transmitted using the LVDS protocol via shielded Ethernet cables, while the RMMs are connected to the DAQ computers with optical Gigabit Ethernet links.

Cosmic trigger primitives are formed from the 29 TFBs on the upstream ECal Super-PØDule based on coincidences between some number of MPPCs. These trigger primitives are transmitted to the CTM and combined with other trigger primitives from the SMRD and the DsECal to create a global cosmic trigger decision when any side pairs of the ND280 detector are traversed by a cosmic ray muon. The MCM receives the accelerator timing signals when a spill happens and transmits trigger as well as periodic clock synchronization signals to the RMMs and TFBs via the SCM. The MCM can also generate pedestal and calibration triggers, such as for synchronous operation of the PØD light injection system. The SCM duplicates most of the functionality of the MCM and allows the configuration and operation of each sub-detector, such as the PØD, in standalone mode independent of the other detectors.

The global ND280 DAQ [2] [13] uses the MIDAS framework [14], developed at TRIUMF, operating on computing nodes running Scientific LINUX operating system. The TFBs are controlled and read out by the front-end processing nodes (FPN), each of which serves two RMMs (3 FPN are used for the PØD). The read-out and configuration is provided by the read-out task (RXT), while the raw data from the TFBs are decoded and formatted for output by the data processing task (DPT). The DPT also performs per-channel histogramming of specific trigger types before zero suppression and inserts the histograms to the output data stream periodically. An event builder process collects the fragments from the sub-detectors, and writes the fully-assembled events to a buffer after basic consistency checks. Finally, an archiver process transfers the completed files to mass storage and creates a preview copy on a local semi-offline system for fast-turnaround calibration and data quality checks.

A global slow control (GSC) complements the global DAQ using the same MIDAS-based software framework. Front-end tasks running on the sub-detector computers connect to various equipment of the sub-detectors and collect monitoring data that are stored in a MySQL database at regular intervals. Any of the variables collected by the GSC can be displayed conveniently through a web interface. Several customized web pages have been developed for controlling and monitoring different components, such as the power supply voltages, TFB internal and external temperatures, the PØD water target levels, etc. Alarms can be set interactively to catch variables out of range and to alert shift personnel.

About 210 TFBs were tested prior to installation in the PØD. The test utilized the internal calibration circuit of the boards, which injects a specific charge into the TFB input channels. The injected signal is provided by a $10 \mathrm{pF}$ capacitor charged to a voltage level up to $5 \mathrm{~V}$, specified by a 12-bit DAC, and then discharged into the selected input channels. The basic functionality of the TFBs was tested by measuring the response of all Trip-t channels at 45 different calibration levels. An RMM emulator board and a simplified version of the MIDAS based DAQ was used to operate the test system and collect the data. The boards with the most uniform gains were selected for installation in the PØD while the others were set aside as spares. The spread of the electronics gain measured in the tests was consistent with the $5 \%$ tolerance of the charge division and calibration capacitors used in the manufacturing of the TFBs.

\subsection{Electronics mounting and cooling}

The power requirements for the 174 TFBs and 6 RMMs in the PØD are listed in Tables 2, 3 and 4. The TFBs were mounted on six rails attached to the outside face of the PØD, as shown in Fig. 3. Each rail consists of two aluminum extruded pipes that contain the cooling water at negative pressure. Aluminum plates, $6.4 \mathrm{~mm} \times 300 \mathrm{~mm} \times 203 \mathrm{~mm}$, were attached directly to the extrusions. The TFBs are thermally mounted to the plates using Dow Corning TP-2160-T4.6-5002 thermal pads. Two TFBs are mounted per plate and there are seven plates of this type in each view. The $y$ coordinate readout has an extra plate with only one TFB mounted. Therefore one rail has a total of 29 mounted TFBs. One RMM services each rail and is mounted on additional aluminum plates attached to the TFB rails. The upstream and central ECals each employ a single rail while the upstream and central water targets each require two.

The voltage requirements of the TFBs including the MPPC voltage are $5.5 \mathrm{~V}, 3.8 \mathrm{~V}, 3.1 \mathrm{~V}, 1.7 \mathrm{~V}$, and $70 \mathrm{~V}(\mathrm{HV})$. Power 
and ground are supplied to the TFBs via six copper bus bars $(3.2 \mathrm{~mm} \times 12.7 \mathrm{~mm}$ for power and $6.4 \mathrm{~mm} \times 12.7 \mathrm{~mm}$ for ground) traversing the full length of the rails. The voltages and ground are supplied by 22 AWG wire coming from the TFB power connector (MOLEX 90142-0020) jumpers to these bars.

\begin{tabular}{|c|c|}
\hline \multicolumn{2}{|c|}{ Power Requirements per TFB } \\
\hline \hline Voltage (V) & Current (A) \\
\hline 1.7 & 0.26 \\
3.1 & 0.60 \\
3.8 & 0.05 \\
5.5 & 0.18 \\
\hline
\end{tabular}

Table 2: Power requirements per TFB. A total of 174 TFBs were needed.

\begin{tabular}{|l||c|c||c|c|c|}
\hline \multicolumn{5}{|c|}{ Power Requirements per Super-PØDule } \\
\hline \hline Super-PØDule & $\begin{array}{c}\# \\
\text { TFB }\end{array}$ & $\begin{array}{c}\# \\
\text { RMM }\end{array}$ & $\begin{array}{c}\text { Vol. } \\
\text { (V) }\end{array}$ & $\begin{array}{c}\text { Cur. } \\
\text { (A) }\end{array}$ & $\begin{array}{c}\text { Power } \\
\text { (W) }\end{array}$ \\
\hline Upstream & \multirow{3}{*}{29} & \multirow{3}{*}{1} & 1.7 & 7.5 & 12.8 \\
ECal & & & 3.1 & 19.9 & 61.7 \\
& & & 3.8 & 4.4 & 16.8 \\
& & & 5.5 & 5.7 & 31.4 \\
\hline Upstream & \multirow{3}{*}{58} & \multirow{2}{*}{2} & 1.7 & 15.0 & 25.6 \\
Water Target & & & 3.1 & 39.8 & 123.4 \\
& & & 3.8 & 8.8 & 33.6 \\
& & & 5.5 & 11.4 & 62.8 \\
\hline Central & \multirow{3}{*}{58} & \multirow{2}{*}{2} & 1.7 & 15.0 & 25.6 \\
Water Target & & & 3.1 & 39.8 & 123.4 \\
& & & 3.8 & 8.8 & 33.6 \\
& & & 5.5 & 11.4 & 62.8 \\
\hline Central & \multirow{2}{*}{29} & \multirow{2}{*}{1} & 1.7 & 7.5 & 12.8 \\
ECal & & & 3.1 & 19.9 & 61.7 \\
& & & 3.8 & 4.4 & 16.8 \\
& & & 5.5 & 5.7 & 31.4 \\
\hline \hline Total & & & & 736.2 \\
\hline
\end{tabular}

Table 3: Power requirements per Super-PøDule

\section{Response Scan of the PØDules}

\subsection{Construction of the PØDule Scanner}

After assembly of each PØDule, the response of each scintillator bar was measured using an automated radioactive source scanner. The goal of the source scan was to measure the position of each bar in the PØDule, to measure variation of response of each bar along its length and to check for dead or otherwise compromised channels.

The scanner was based on a $9^{\prime} \times 10^{\prime}$ custom $\mathrm{CNC}$ router manufactured by ShopBot of Durham, North Carolina. To convert it
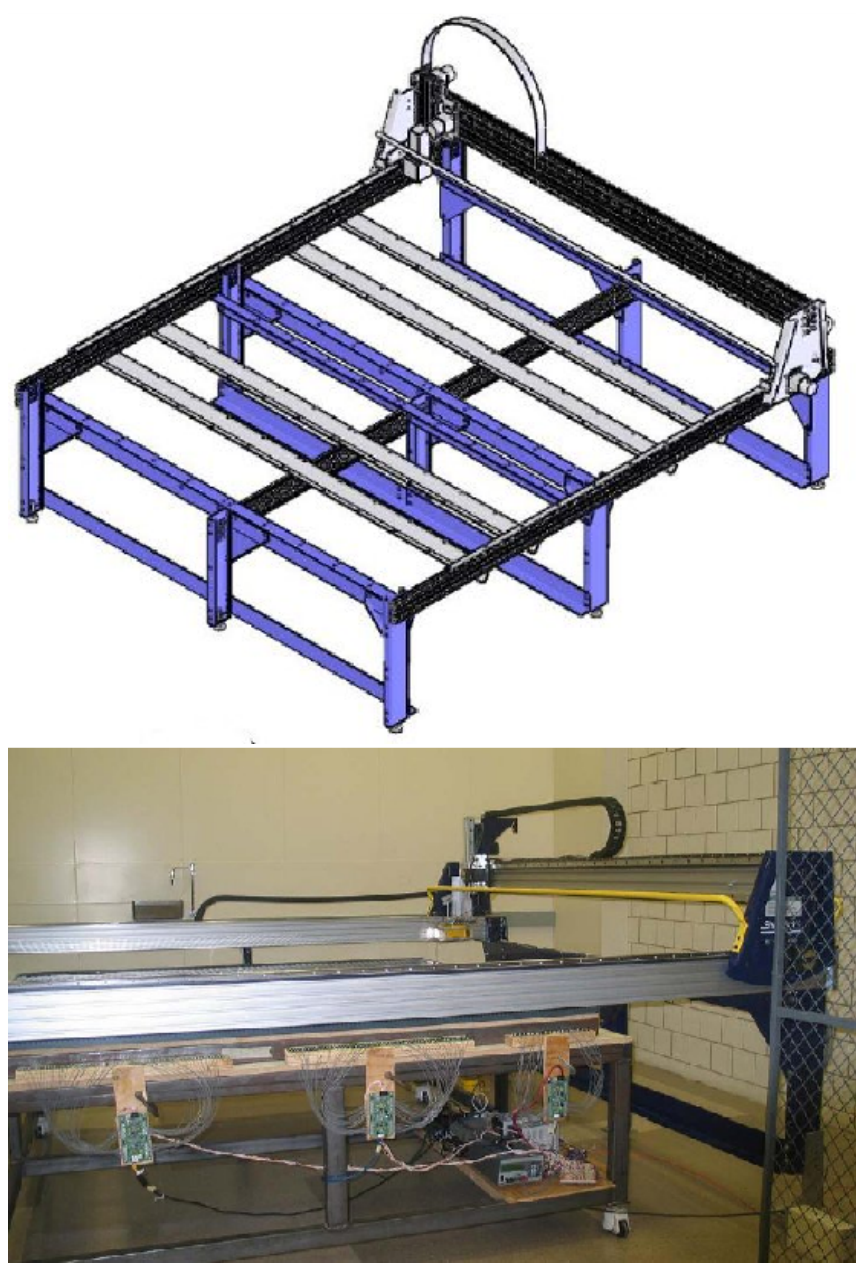

Figure 13: An engineering schematic (left) of the custom CNC router modified to serve as the PØD scanner (right), shown with a PØDule that is ready to be scanned. 


\begin{tabular}{|c|c|}
\hline \multicolumn{2}{|c|}{ Power Requirements per RMM } \\
\hline \hline Voltage(V) & Current(A) \\
\hline 3.1 & 0.37 \\
3.8 & 2.88 \\
5.5 & 2.44 \\
\hline
\end{tabular}

Table 4: Power requirements per RMM. A total of 6 RMM were needed.

for use as a scanner, the spindle router was replaced with a unit consisting of a radioactive source, a video camera for alignment of the PØDule to the router table coordinates, and a depth sensor to measure distance from the source to the surface of the PØDule. The source was a $0.7 \mathrm{mCi}{ }^{60} \mathrm{Co}$ source housed inside a 4 " thick lead shield. Inside the shield, the source was located in a 0.27 " diameter bore hole, 1.75 " from the bottom surface, and collimation of the source on the PØDule was achieved by cutting away the lead in a fixed cone with the curved surface angled $22.7^{\circ}$ from the vertical. Figure 13 shows the scanner with a PØDule ready to be scanned.

The scanner motion was controlled by a LabView based program to drive the source to predetermined locations so that a current in the photosensor due to the source could be measured on each channel. For each of the two views, the source was moved perpendicular to the bar direction in $1.7 \mathrm{~cm}$ steps. Since the beam from the source was much wider than the step size, response as a function of position could determine both the location of the strip and its response to the source at that point. Eleven such scans were performed at different positions along the strips to measure the change in response along the bar.

\subsection{Operation of the Scanner}

Each scan was conducted in $1.7 \mathrm{~cm}$ steps along eleven equally spaced lines for both $x$ and $y$ coordinates. The PØDule corners were used as reference points for the scanning and viewed using a web camera mounted on the moving scanner element next to the source shielding. The scanner control and DAQ computer provided the coordinates for the next scanning position in addition to collecting and processing the data. The same set of readout electronics (See Section 5) was used for all scanning operations.

\subsection{Analysis of Scanner Data}

The manufacturer-suggested bias voltage values were used for each sensor without any additional tuning. Since a gamma radioactive source was used for the scanning process, a random trigger was used. First, the dark noise spectrum was obtained for the MPPC sensors without the source, which established the pedestal and provided an independent check of the MPPCs. A channel exposed to the source experiences a pedestal shift to lower values proportional to the exposure. Thus the channels with source signal will have the pedestal at lower ADC counts than without it. It was expected that the 11 points measured along each bar should lie on an approximately exponentially decaying smooth curve corresponding to the light attenuation in a WLS fiber [15] with a mirrored end. A sample of the scanner

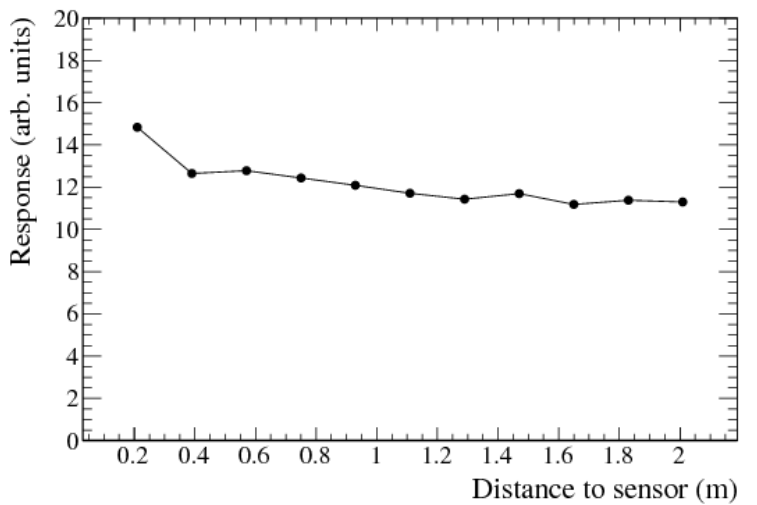

Figure 14: Typical scanner response of a single scintillator bar.

output for a single scintillator bar is given in Figure 14. A sharp step in this curve would indicate damage along the optical fiber. Only two such fibers were discovered (and replaced), out of 10,400 PØD channels.

Additionally, the spread of the channel outputs was obtained. For example, for the $x$ PØDule bars, as the scanner moved along the $x$-axis with a fixed $y$ position, the output of each channel was recorded as a function of $x$. For each bar this resulted in a constant pedestal value with a peak when the scanner reached the $x$ position of that bar. A Gaussian was fit to that peak, to determine the response of the bar at that $y$ position. This was then repeated for a number of different $y$ positions along the bar.

The bar response was fit as a function of position along the bar to a double exponential attenuation, motivated by the known two dominant WLS emission peaks, plus a reflection term, namely

$$
N \times\left[f e^{\frac{-y}{L}}+(1-f) e^{\frac{-y}{S}}+R\left(f e^{\frac{-(2 b-y)}{L}}+(1-f) e^{\frac{-(2 b-y)}{S}}\right)\right],
$$

where $N$ is the overall normalization, $f$ is the fraction of light in the long mode, $L$ is the long attenuation length, $S$ is the short attenuation length, $R$ is the reflectivity, and $b$ is the length of the bar. Here only $L$ and $N$ were allowed to vary in the fit, while the other parameters were held at fixed values. A similar procedure was also applied to $y$ PØDule bars. The corrected response and the long attenuation length of each bar are plotted in Figure 15. Note that the $y$ bars were on the top of the scanned PØDules, so the average response was lower in the $x$ bars due to the absorption of the source.

\section{Pre-installation Testing}

\subsection{Channel Checks using Dark Noise}

The dark noise of the MPPC detectors makes a good parameter for a quick check of the photosensor and the readout electronics channel. The test setup consisted of three back-end boards: a local Master Clock Module (MCM), which provided a synchronized clock to the other back-end boards and controlled the data taking; an RMM connected to all of the mounted TFBs; and a cosmic trigger module (CTM). For the ECal SuperPØDules, each TFB was also connected to the CTM, separated 

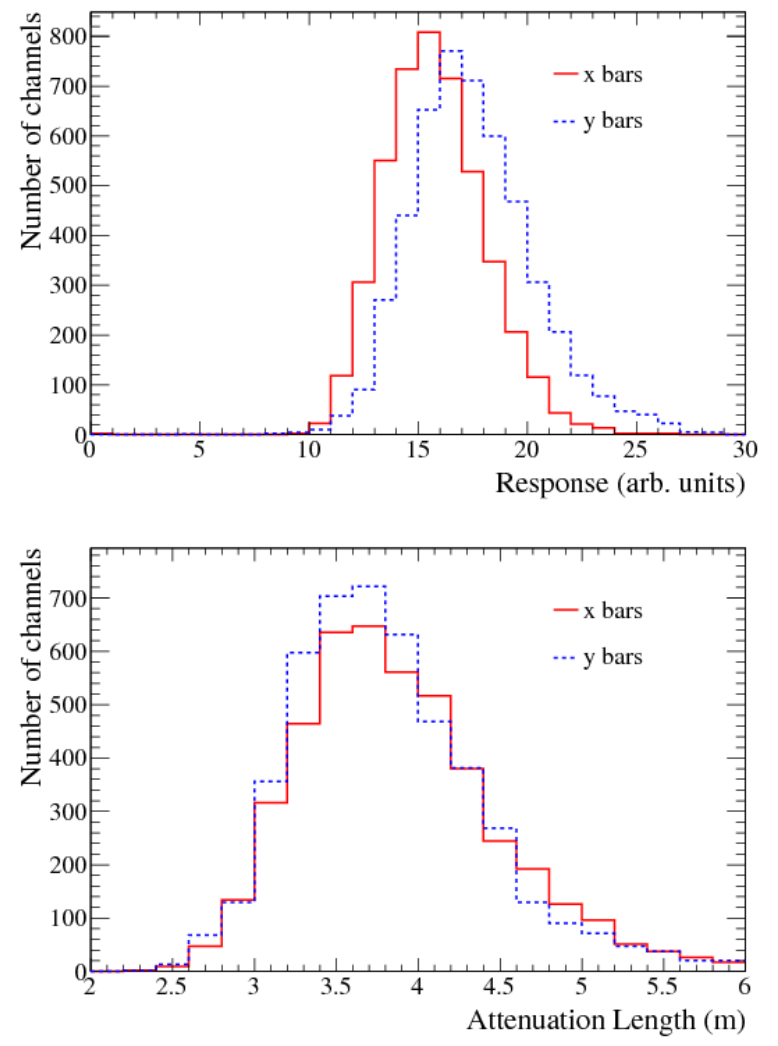

Figure 15: Corrected response ( $N$ in Equation 1 for both $x$ and $y$ bars (top); Long attenuation length ( $L$ in Equation 1 for both $x$ and $y$ bars (bottom).

into four groups of primitives (upstream $x$, downstream $x$, upstream $y$ and downstream $y$ ).

The pre-installation check of all PØD channels into the ND280 detector was carried out in two steps. The first step included dark noise testing that took place during the scanning. The results of the dark noise test were thus indicative of the actual MPPC performance after their installation into the detector modules. 14 MPPCs were found to be outside our selection criteria and had to be replaced at this stage. The typical bad sensor signature was either no output (no pedestal or no clearly separated p.e. peaks on the response histogram), or an output with barely separable p.e. peaks. The testing was done at the manufacturer recommended biasing voltage values for each sensor, which results in a gain of around $6.5 \times 10^{5}$ at a photosensor temperature of $25^{\circ} \mathrm{C}$.

The second step for this pre-installation check was performed after all the TFB boards were installed on the sides of each of the four Super-PØDules and had been shipped to the JPARC facility. A separate readout setup, including a portable RMM module, power supplies, and DAQ computer was connected to each TFB board. The dark noise histograms from a random trigger were plotted for each channel.

Dark noise checks resulted in three TFBs and two MPPCs being replaced due to anomalous dark noise rates. A second-pass of dark noise testing was undertaken after installation of the water system level sensors. This was deemed necessary since some electronics had been moved to allow access to the water target region. The check revealed that several coaxial cables between the MPPCs and the TFB boards had to be reconnected or replaced. After this was completed, all 10,400 PØD channels were operational.

\subsection{Checkout with the Light Injection System}

Prior to installation of the PØD into the ND280 basket, the light injection system was used to confirm that the fiber to photosensor to TFB to RMM detection chain was correct functioning.

The Light Injection System (LIS) was flashed at two amplitudes: a lower amplitude to test the system response in a region where most of the channels were read out from the high gain ADC and a higher amplitude to test the response of the low gain ADC.

The LIS allowed the identification of production defects that could be repaired before PØD installation. One method utilized was to look at the measured signal on all fibers within a single PØDule illuminated by a single LIS pulser channel. The overall features of this distribution are understood by the LIS cavity geometry and the position of the fibers with respect to the light source. Anomalous signals on a fiber may indicate a production defect such as a misplaced fiber guide.

\subsection{Checkout using Cosmic Rays}

During the PØD checkout prior to installation in the experimental hall, cosmic ray data were taken with the full data acquisition chain and the full software chain. A detailed description of this work can be found in [16].

Each Super-PØDule was tested independently, with the mounted TFBs connected to the setup described in 7.1.

In the cosmic ray muon mode, a TFB with integrated charge over threshold produces a signal sent to the CTM, which triggers the RMM to read out all of the TFBs if at least one board in each primitive group (upstream vertical bars, downstream vertical bars, upstream horizontal bars and downstream horizontal bars) produced a signal to ensure that the cosmic had passed through the Super-PØDule and produced hits in both views.

The cosmic data was calibrated using the dark noise data and passed to the PØD reconstruction software. Integration cycles with more than 20 hits (a normally incident muon produces $\sim 28$ hits), were searched for muon tracks. Events with a single track were selected and used for the first minimum-ionizing particle (MIP) light yield study (Section 8.2.

Figure 16 shows the residuals of the reconstructed tracks in the Upstream ECal, which have been fit to a Gaussian distribution. The fitted parameters of $\mu_{x, y}=0.0 \mathrm{~mm}, \sigma_{x}=2.8 \mathrm{~mm}$ and $\sigma_{y}=3.2 \mathrm{~mm}$, gave the first direct measurement of the PØD's tracking resolution.

\section{Detector Calibration and Performance}

\subsection{Gain Calibration}

The low level charge calibration converts raw ADC response of the electronics to photoelectron units. It is performed in three 

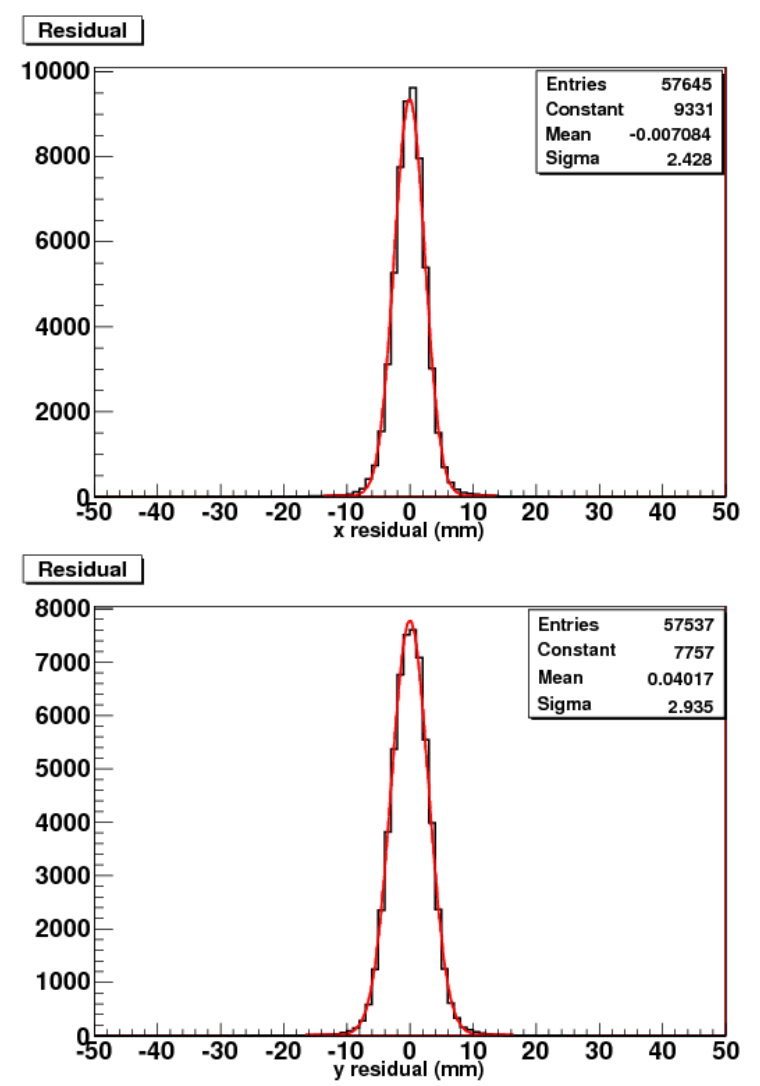

Figure 16: Residual distributions of reconstructed 3D tracks (solid histogram) in the $x z$ (left) and $y z$ (right) planes. The distributions are fit to a Gaussian distribution (solid curve).

stpdf: pedestal subtraction, correction for the electronics nonlinearity and the relative low/high gain response, and correction for the MPPC gain variations.

The pedestal, i.e. the baseline response of the MPPC and electronics without input signal, as well as the MPPC gain, is measured using non zero-suppressed dark rate noise (Figure 17). The pedestal peak in the dark noise spectrum is fit to a Gaussian function in each integration cycle separately to account for the small variations among the cycles. The mean of the Gaussian gives the pedestal constant used for the pedestal subtraction. The MPPC gain is measured as the separation between the pedestal and the 1 p.e. peak after combining the dark noise spectra from all 23 integration cycles which were corrected for individual pedestal shifts. The two peaks are fit to a double Gaussian and the difference in their means is used to measure the photoelectron unit in ADC values. Since the MPPC overvoltage, as well as the pedestal, is quite sensitive to the temperature at a fixed bias voltage, the gain and pedestal require continuous monitoring and updating.

Before converting the signal into photoelectron units, the raw ADC response needs to be corrected for the non-linearity of the electronics and the relative gain difference between the high and low gain response. The response of each input channel is measured using the internal TFB calibration circuit as a series of 174 signal levels that covers both the high and low gain dynamic range. This measurement is performed in situ when the

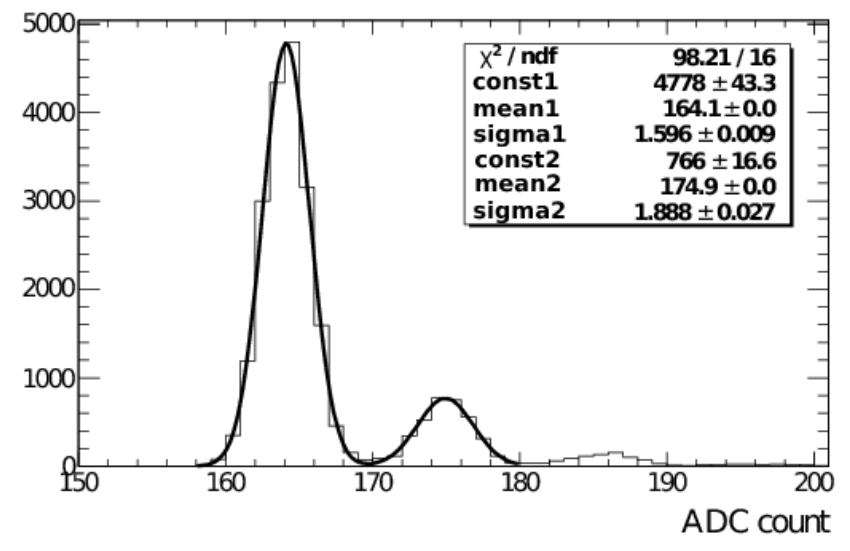

Figure 17: Typical digitized dark noise spectrum of an MPPC with a double Gaussian function fitted to the pedestal and 1 p.e. peaks.

MPPCs are powered since the capacitance of the photosensors and the mini-coax cables connecting the sensors to TFBs represent a significant additional capacitance on the input, altering the effective electronics gain. The measured response as a function of the calibration level is fit to a bi-cubic polynomial with nine free parameters. This parametrization is used to correct the raw ADC values during the offline calibration of the data. The bi-cubic function allows an adequate representation of the Trip-t non-linearity with residuals typically smaller than a few percent (Figure 18). The electronics gain and non-linearity are fairly stable, requiring only occasional checks, and therefore the constants are updated only if there is a hardware change to the front-end electronics.

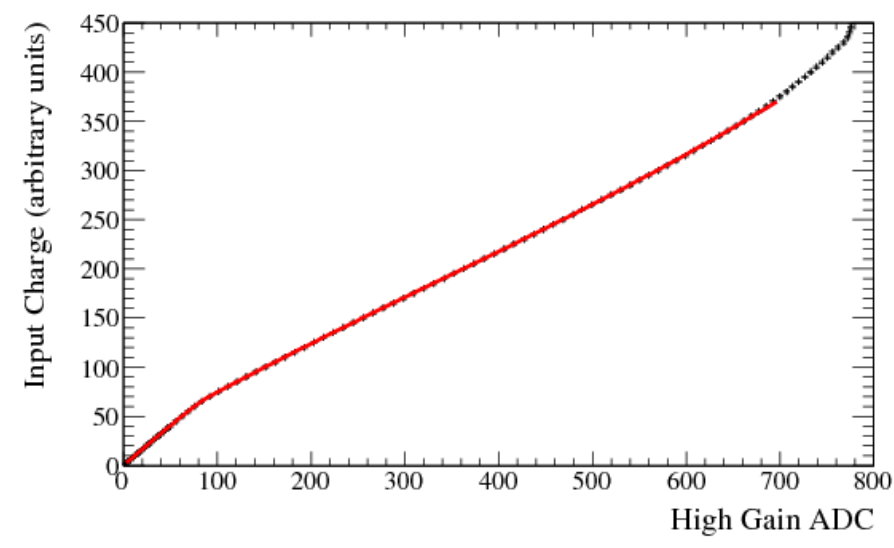

Figure 18: Charge versus ADC for a high-gain Trip-t channel fit to a bi-cubic function.

\subsection{MIP Light Yield}

A useful characteristic of minimum ionizing particles (MIPs) is that their energy loss is only weakly dependent on their energy. Therefore, for high energy muons passing through the detector, the mean energy deposition per unit length is a constant. With a sample of through-going muons, this constant can be determined. The first sample used was the cosmic checkout 
data, selecting tracks with $\cos \theta>0.8$ [16] (where $\theta$ is the angle that the track makes with the $z$ axis of the detector) but this was for individual SuperPØDules only.

After the PØD was installed in the basket, it became possible to calibrate all PØDules with the same data sample. The best sample was through-going muons from beam neutrino interactions in the wall or sand and rock upstream of the PØD. After reconstruction, events were selected with a single $3 \mathrm{D}$ track entering the front face of the $\mathrm{P} \varnothing \mathrm{D}$ and exiting out the downstream end.

These events were analyzed to show each layer's detection efficiency. Due to the triangular design of the PØD's scintillator bars, a normally incident MIP is most likely to pass through two bars, as demonstrated in Fig. 19 . However, depending on the path taken, there is a chance that one bar is untouched, or that the signal is below the noise threshold cut applied by the reconstruction.

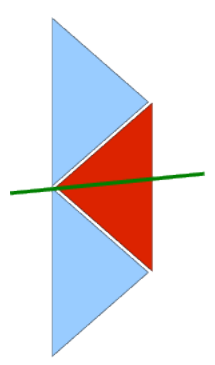

Singlet

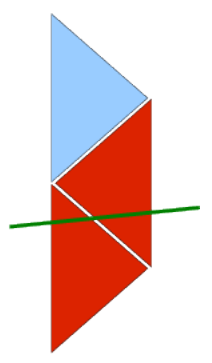

Doublet
Figure 19: Illustration of a singlet and doublet, as a MIP passes through a PØDule layer.

The results, shown in Fig. 20 indicate the probability of finding 0,1 or 2 hits in each $x$ or $y$ plane. The tracking efficiency is $100 \%$ for all but the first three scintillator planes, which is explained by the selection criteria allowing a small number of first layer neutrino interactions into the sample.
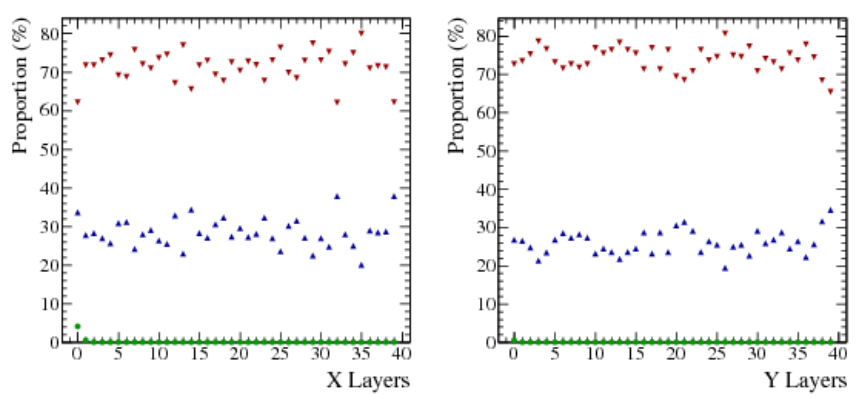

Figure 20: The PØD layer detection efficiency. The plots show the proportion of tracks with 0 (green circles), 1 (blue triangles) and 2 or more (red inverted triangles) hits in a layer, for both $x$ and $y$. The small excess of 0 hits in the upstream $x$ layer is due to neutrino interactions in the first $y$ layer passing the cuts.

Figure 21 shows the summed charge deposit for the two hit sample, after calibration and path correction using the reconstructed track angle. The plot has been fit with a GaussianLandau distribution, and returns a most probable value of 37.9 p.e./mip/cm. This value provides a known point, which each channel of the PØD can be calibrated to, ensuring a constant response for the detector.

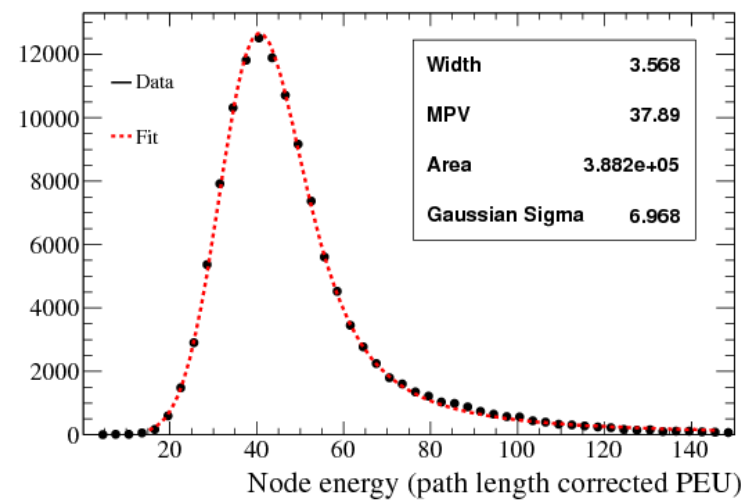

Figure 21: The summed charge deposit for doublets from through-going muons originating from neutrino interactions outside the detector. The fit is a Gaussian-Landau distribution.

\subsection{LIS Operation and Performance}

The LIS system simultaneously illuminates the entire PØD and is read out at in bursts of $20 \mathrm{~Hz}$ interspersed with other trigger types. The current settings give the LIS system an effective rate of $1.5 \mathrm{~Hz}$. The LIS system cycles through a set of ten amplitudes, each with 500 flashes, taking about one hour for a complete cycle. Figure 22 shows the average ADC signal produced by each of the four pulser boxes during a typical run. Each plateau corresponds to a single amplitude. The sequence of amplitude was purposefully chosen to produce a clear step structure in the response to enable easy visual separation of the groups from each other.

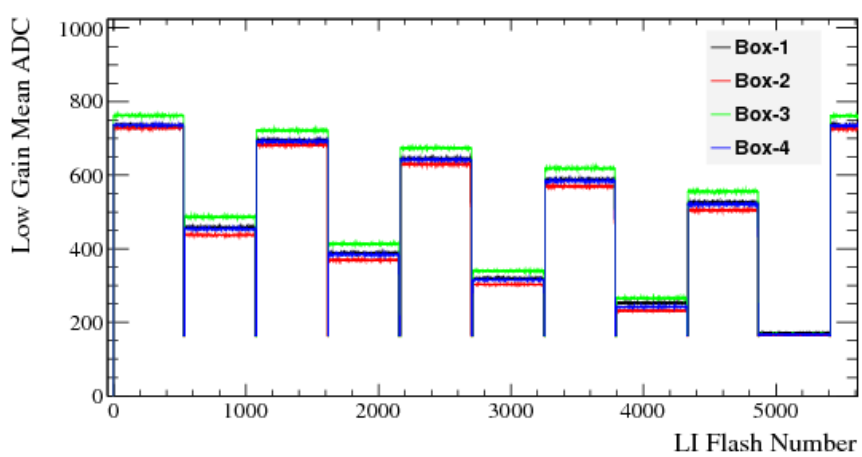

Figure 22: Average signal produced by each pulser for a full cycle of 10 amplitudes.

Besides providing the ability to quickly determine the correct functioning of all PØD photosensors, the LIS provides a tool to monitor the stability of the photosensor signal, shown for a portion of a physics run is shown in Fig. 23. The variation over short periods of time can be attributed to changes in the photosensor gain. Shifts that are different with respect to each pulser can be evidence for malfunctions in the PØD readout electronics. 


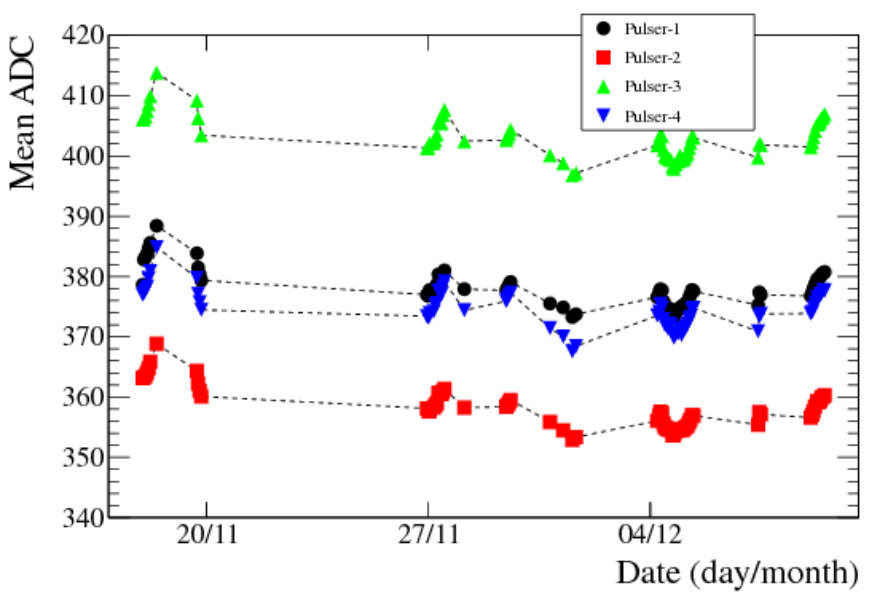

Figure 23: PØD stability for a portion of a physics run showing the average signal in all photosensors illuminated by the LIS system. Note the suppressed zero.

\subsection{Water Target Filling and Monitoring}

The depth sensors were found to have fluctuations of $\pm 1 \mathrm{~mm}$ but had a $\pm 15 \mathrm{~mm}$ calibration offset before insertion into the PØD. This offset was reduced by using the fixed binary wet-dry level sensors to provide calibration reference points in situ. We expected the water level to drop in some layers due to deflection of the plastic scintillator. As shown in Fig. 24 the largest change in water level is closest to the downstream end of the PØD which is not directly supported by the basket.

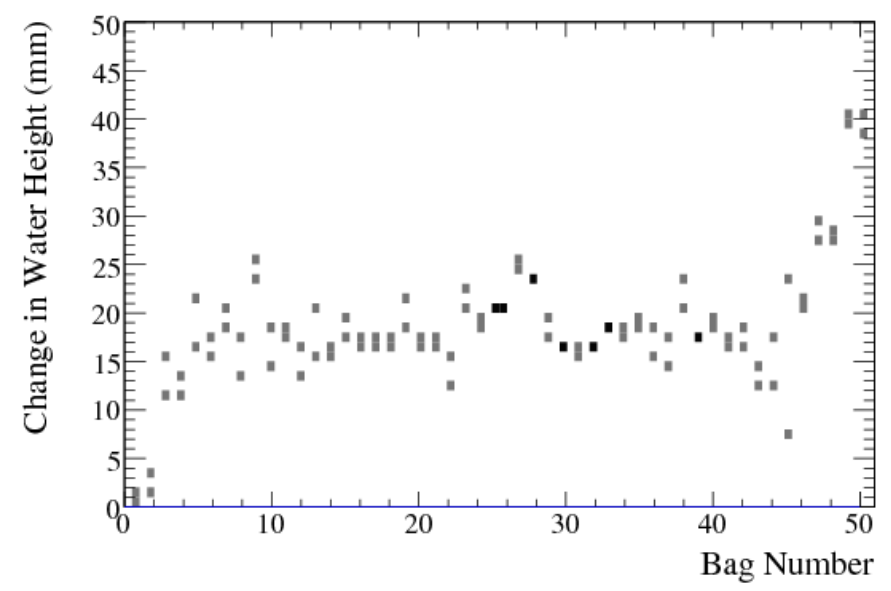

Figure 24: Water level shift after 19 days (2 sensors per bag).

Geometry and the measured dimensions of the PØD constrain the uncertainty on the total mass of water in the fiducial volume to approximately $3 \%$. The addition of measurements from the WL400 depth sensors and the external tank volume measurements reduce this uncertainty to less than $1 \%$.

\section{Conclusion}

The PØD subdetector in the T2K near detector will be used to meet the $\mathrm{T} 2 \mathrm{~K}$ physics goals by providing a determination of the neutral current $\pi^{0}$ rate for the T2K neutrino beam on a water target. Analyses on this measurement and several others are underway.

The detector was installed in October 2009 and has been taking neutrino beam data since January 2010. To date, there have been no major problems and the PØD is performing as expected.

\section{Acknowledgements}

The PØD detector has been built and operated using funds provided by the U.S. Department of Energy. In addition, the participation of individual researchers and institutions in the construction of the PØD has been further supported by funds from the U.S. Department of Energy Early Career Program and from the City University of New York PSC-CUNY Research Award Program. The authors also wish to acknowledge the support provided by the collaborating institutions, particularly the State University of New York at Stony Brook, Office of the Vice President for Research and finally to thank our T2K colleagues for their invaluable help during the installation and commissioning of the detector.

\section{References}

[1] Y. Fukuda, et al., Nucl. Instrum. Meth. A501 (2003) 418-462.

[2] K. Abe, et al., Nucl. Instrum. Meth. A659 (2011) 106-135.

[3] M. Yokoyama, et al., Nucl. Instrum. Meth. A622 (2010) 567-573.

[4] A. Dyshkant, V. Rykalin, A. Pla-Dalmau, D. Beznosko, AIP Conf. Proc. 867 (2006) 513-520.

[5] D. Drakoulakos, et al., FERMILAB-PROPOSAL-0938 (2004).

[6] K. S. McFarland, Nucl. Phys. Proc. Suppl. 159 (2006) 107-112.

[7] A. Pla-Dalmau, A. D. Bross, V. V. Rykalin, B. M. Wood, FERMILABCONF-05-506-E (2005). Proceedings of 2005 IEEE Nuclear Science Symposium and Medical Imaging Conference, Puerto Rico, 23-29 Oct 2005 .

[8] A. Vacheret, et al., Nucl. Instrum. Meth. A656 (2011) 68-83.

[9] P. Adamson, et al., Nucl. Instrum. Meth. A521 (2004) 361-366.

[10] J. Abraham, et al., Nucl. Instrum. Meth. A523 (2004) 50-95.

[11] A. Vacheret, S. Greenwood, M. Noy, M. Raymond, A. Weber, Nuclear Science Symposium Conference Record, NSS '07 (2007) 1984-1991.

[12] L. Bellantoni, P. Rubinov, D0 note 4845 (2005).

[13] M. Thorpe, et al., IEEE Trans. Nucl. Sci. 58 (2011) 1800-1806.

[14] S. Ritt, P. Amaudruz, K. Olchanski, MIDAS (Maximum Integration Data Acquisition System), 2001. http://midas.psi.ch.

[15] D. Beznosko, A. Dyshkant, C. Jung, C. McGrew, A. Pla-Dalmau, et al., FERMILAB-FN-0796 (2007).

[16] P. T. Le, Ph.D. thesis, State University of New York at Stony Brook, 2009. 